\title{
Fabry-Perot measurements of barium temperature in fluorescent lamps
}

\author{
S Hadrath \\ OSRAM GmbH, Research Europe, SIGMA Technopark Augsburg, Werner-von- \\ Siemens-Str. 6, 86159 Augsburg, Germany \\ R Garner \\ OSRAM Sylvania, Central Research and Services Laboratory, Beverly, MA \\ 01915-1015, USA \\ E-mail: stefan.hadrath@ osram.com
}

\begin{abstract}
A scanning Fabry-Perot interferometer (FPI) is used to determine the temperature of barium atoms that are liberated from the electrodes of fluorescent lamps during their steady-state operation. Barium, a constituent of the work function lowering emitter material that is placed on the tungsten coil that forms the electrode, is liberated primarily by evaporation from the hot $\left(\sim 1300^{\circ} \mathrm{K}\right)$ thermionic electrode. However, there may be situations or modes of operation in which barium is, in addition, sputtered, a condition which may lead to increased end-darkening, shortened life, and increased mercury consumption in the lamp. Using the FPI diagnostic, the occurrence of sputtering is inferred when barium temperatures are much greater than the electrode temperature. The FPI diagnostic senses resonance radiation $(\lambda=553 \mathrm{~nm})$ emitted by barium atoms excited in the low pressure discharge environment, and infers temperature from the Doppler broadened linewidth. The diagnostic has proven to be successful in a number of situations. Measurements have been made on rare gas discharges and on $\mathrm{Hg}$-argon discharges for different discharge currents, gas pressures, and auxiliary coil currents. Measurements are phase resolved for a.c. driven discharges.
\end{abstract}

PACS numbers: $52.70 . \mathrm{Kz}, 52.80 .-2,52.75 . \mathrm{Xx}$,

Submitted to: J. Phys. D: Appl. Phys. 


\section{Introduction}

It is well known that the end-of-life of a fluorescent lamp typically occurs when at least one of its electrodes ceases to function properly. Although the precise details of the electrode's state at end-of-life are not fully known, it is generally understood that an electrode's ultimate demise is due to the cumulative loss of the work function-reducing emitter material which occurs over the course of lamp life. The emitter material, which is situated on and within the windings of a tungsten coil, is comprised most commonly of a mixture of three alkaline earth oxides, $\mathrm{BaO}, \mathrm{SrO}$ and $\mathrm{CaO}$, plus a small amount of $\mathrm{ZrO}_{2}$. Although all atomic species that are present in the emitter are to some degree lost from the electrode ${ }^{*}$, the loss rate of barium is the greatest, at least during the bulk of lamp life. Therefore, the monitoring of barium liberated from the electrode (atomic and/or ionic) perhaps provides the best opportunity for assessing the state and performance of the electrode within an operating lamp.

Loss of emitter material, principally barium, is an inevitable consequence of the electrode's normal operation, and is intimately tied to the nature of its interaction with the discharge [2]. Evaporation and sputtering are the two predominant loss mechanisms. Evaporation occurs almost exclusively during steady-state lamp operation, during which the electrode emits electrons into the discharge through the process of thermionic emission. The temperature necessary for sustaining such emission is in the range of about $1300-1400^{\circ} \mathrm{K}$, and this inevitably leads to an evaporation rate which, in the absence of other loss mechanisms (the ideal situation), allows the lamp to operate for some tens of thousands of hours.

Loss of emitter material by sputtering is very intense during the starting period of a lamp, particularly in cases where the electrode is initially at room temperature (so-called 'instant start'). In these situations, the initial state of the lamp discharge, after the ignition process, is that of a glow discharge, whereby the electrons are emitted from the electrode through the process of secondary electron emission induced by bombardment of high energy ions from the plasma. In order for this process to be effective in supplying the necessary electron flux, sheath potentials during the cathode phase (i.e., cathode falls) of several hundred volts must develop. The energy that this imparts to the bombarding ions leads to high sputter yields, possibly on the order of 0.1 [2]. Straightforward optical absorption techniques have been used to sense the plethora of barium atoms liberated during the glow discharge phase, and line-averaged densities are easily inferred from these measurements [3,4]. The glow discharge phase of an instant start lamp may last several tens of milliseconds, terminating with a relatively quick transition to the thermionic arc when the electrode has been heated by the bombarding ions to sufficiently high temperatures.

It is generally assumed that very little, if any, sputtering of barium occurs during the steady-state thermionic arc of a typically-operating lamp [2] $]^{\dagger}$. It is presumed that most barium is lost simply by evaporation during this time. However, values of sheath potential during the cathode phase of the lamp may reach $\sim 13-15$ Volts $[5,6]$, which is near the threshold for the sputtering process to occur. The threshold for sputtering is not precisely known, but it is believed to be about 16 Volts

\footnotetext{
*All atomic species of the emitter (Ba, Sr, $\mathrm{Ca}, \mathrm{O}, \mathrm{Zr}$ ), and also tungsten from the coil, have been found on the walls and other internal structures (e.g. shields, electrode support posts) within lamps [1].

'A 'typically-operating lamp' is, for example, the ubiquitous linear T8 (1 inch diameter) lamp which, in the North American market, has about 2.5 Torr argon buffer gas and operates with $180 \mathrm{~mA}$ rms steady-state discharge current.
} 
[7]. ${ }^{\ddagger}$ Ideally, the sheath potential during the entire phase of the lamp would be less than the threshold, and this is perhaps the case for typically-operating lamps. But, since the threshold is not precisely known, and since there may be uncertainties in cathode fall measurements on the order of a volt, the presumption of zero sputtering might be incorrect.

It is well known that sputter yields increase in exponential fashion as energy of the incident ion increases above the threshold value [8]. Therefore, even small deviations from typical lamp conditions may increase the cathode fall just enough to drastically increase sputter yield, perhaps to levels where it is comparable to, or even greater than, evaporation. Such deviations may be due to non-standard operating conditions. For example, it is well known that cathode fall increases with decreasing gas pressure and also with decreasing discharge current. The latter is often compensated for by the addition of external ohmic heating of the electrode, which increases hot spot temperature without the need for the increased cathode falls. Furthermore, unintended issues during the manufacture of the lamp may lead ultimately to greater cathode falls in the finished lamp, perhaps due to higher work function. The manufacturing process is susceptible to such issues, particularly during the electrode breakdown/activation phase, during which the compounds that are coated on the electrode, a triple-carbonate of $\mathrm{BaCO}_{3}, \mathrm{SrCO}_{3}$ and $\mathrm{CaCO}_{3}$, are chemically converted to the corresponding oxides.

The occurrence, or enhancement, of sputtering during the thermionic arc may lead to undesirable effects, such as severe end darkening, diminished lamp life, and increased mercury consumption [1]. Therefore, it is of interest to ascertain if, and under what conditions, sputtering may occur. To this end, we have developed an optical-based diagnostic which we have subsequently used to investigate the effective temperature of the barium liberated from the electrode during steadystate lamp operation. The diagnostic senses the emission of the barium resonance line at $553 \mathrm{~nm}$, and incorporates a tunable Fabry-Perot interferometer (FPI) that is capable of spectrally resolving the line. Since the line is Doppler broadened in the low pressure discharge environment of a fluorescent lamp, the effective temperature of the barium can be deduced. The underlying idea is that the (effective) temperature of sputtered barium is higher than the temperature of evaporated barium. The diagnostic has proven to be successful in a number of situations. Measurements have been made on rare gas discharges and on $\mathrm{Hg}$-argon discharges for different discharge currents, gas pressures, and auxiliary coil currents. Measurements are phase resolved for a.c. driven discharges. The diagnostic is described here, and the results and interpretations of the measurements are presented.

The diagnostic was inspired by previous work in which the effective barium temperature during the glow discharge (starting) phase of an instant start lamp was measured by laser absorption [9]. In that work, a single mode, tunable diode laser was utilized, which operated over a $\sim 100 \mathrm{GHz}$ spectral range in the vicinity of $649.8762 \mathrm{~nm}$, corresponding to the barium transition $6 \mathrm{~s} 5 \mathrm{~d}^{3} \mathrm{D}_{3} \rightarrow$ $5 \mathrm{~d} 6 \mathrm{p}^{3} \mathrm{D}_{3}{ }^{0}$ (lower level $\sim 1.14 \mathrm{eV}$ above the ground state). Some results from that work are presented here. It was found that typical effective temperatures of barium were on the order of $2000^{\circ} \mathrm{K}$ over the entire period of the start. During this time the electrode is near room temperature and only slowly heats up, achieving thermionic temperatures in a sudden transition at the end of the starting regime.

\footnotetext{
* The threshold has presumably been inferred from the so-called disintegration voltages (20-25Volts) of mercury and rare gas plasma-based tubes which are the subject of the reference.
} 


\section{Description of Experiment}

\subsection{Optical setup}

Figure 1 shows a diagram of the optical setup. The hot spot (plasma attachment) region of an electrode of an operating lamp is imaged by an achromatic lens (L1) with focal length $f_{1}=100$ $\mathrm{mm}$ to a circular pinhole (A1) with diameter $d_{p i n}=100 \mu \mathrm{m}$. The hot spot and its image are located $\sim 200 \mathrm{~mm}$ in front of and behind the lens, respectively, resulting in $\times 1$ magnification. Thus, the shape and size of the region from whence radiation is measured is approximately the same as that of the pinhole. The barium emission decreases sharply (on the order of millimeters) with distance from the hot spot [4]. Therefore, most of the radiation that is sensed is essentially that which is emitted from the discharge just in front of the hot spot.

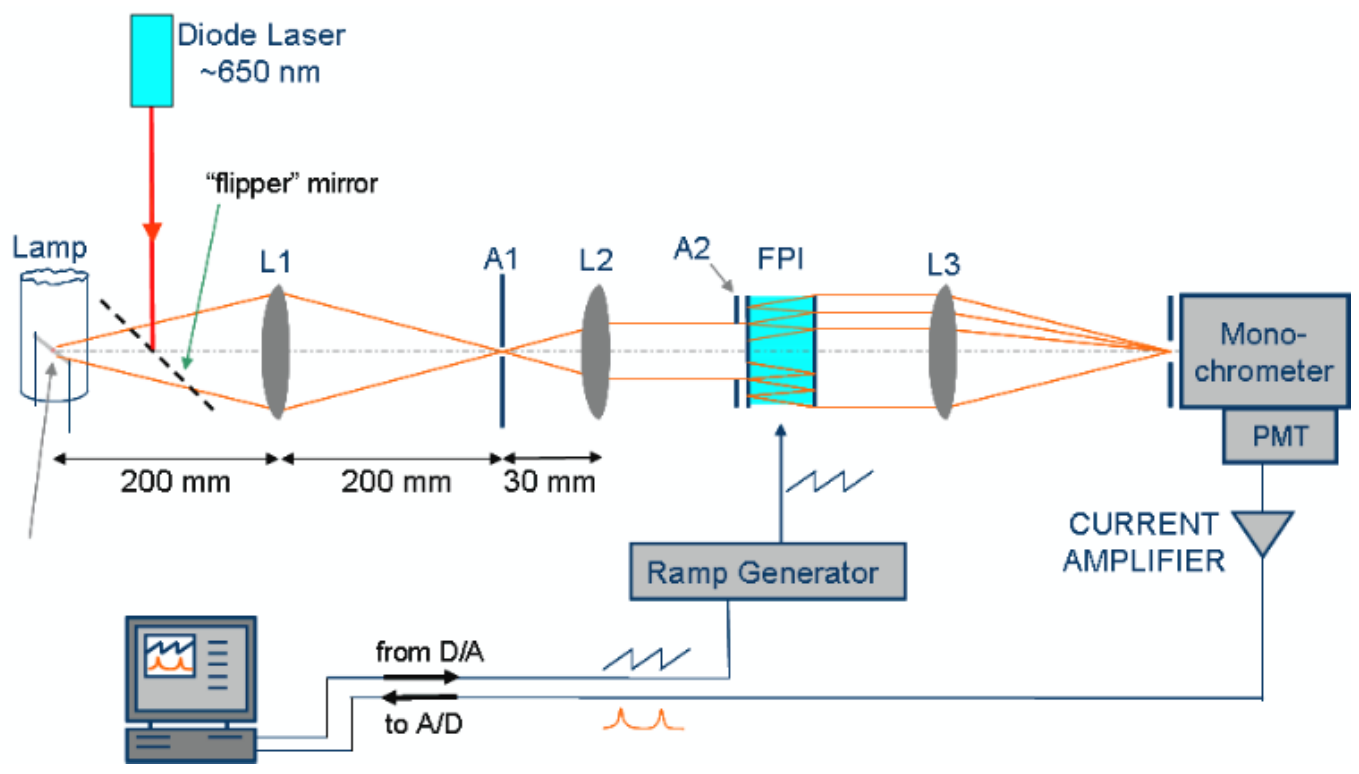

Figure 1. Optical setup with fluorescent lamp FL, Fabry-Perot interferometer FPI (EXFO, TL-0015), achromatic lenses L1, L3 ( $\mathrm{f}=100 \mathrm{~mm}, \varnothing=25 \mathrm{~mm}$ ) and L2 ( $\varnothing=30 \mathrm{~mm}, \varnothing=25 \mathrm{~mm}$ ), grating monochromator, photomultiplier tube PMT, circular apertures A1 $(\varnothing=100 \mu \mathrm{m})$ and A2 $(\varnothing=12 \mathrm{~mm})$, amplifier Amp (Stanford Research SR570), ramp generator RG (EXFO RG-93), and a combined digital-toanalog (D/A) and analog-to-digital (A/D) board (Data Translation DT3010), located inside the computer.

The pinhole (A1) is situated in the focal plane of, and coaxial with, the next lens (L2), an achromat with focal length $f_{2}=30 \mathrm{~mm}$, and this is followed by the FPI. The configuration of pinhole and lens L2 establishes the maximum angle of incidence $\left(\theta_{m}=d_{\text {pin }} / 2 f_{2} \approx 1.67 \mathrm{mrad}\right)$ of the radiation that is incident at the FPI (assuming geometric optics with the para-axial approximation). Because of the non-point-like nature of the measurement region, angular spread of the radiation at the FPI entrance aperture is unavoidable. A smaller angular spread would of course be desirable, but this would come at the expense of reduced signal. The present setup represents a compromise between these two issues. Detailed discussion of the analysis and interpretation of data, given the existence of angular spread, is given in Section 3. 
An adjustable circular aperture (A2) is situated at the entrance of the FPI to provide the capability of reducing the entrance aperture diameter. This allows for improved FPI performance due to increased finesse.

Radiation exiting the FPI is focused by an achromatic lens (L3) with focal length $f_{3}=100 \mathrm{~mm}$ onto the entrance slit of a $0.5 \mathrm{~m}$ monochromator. The radiation passing through is sensed with a photomultiplier tube (PMT) behind the exit slit. The monochromator is used as a rough wavelength selector of the barium line of interest at $553 \mathrm{~nm}$. Of course, with this setup it is straightforward to sense other emission lines as well, although it is noted that the mirror coatings of the FPI are optimized for $553 \mathrm{~nm}$. The PMT output signal is routed to a current amplifier (Stanford Research SR570). Typical (peak) photocurrents from emission measurements from fluorescent lamps are tens of nA.

The Fabry-Perot interferometer (EXFO TL-0015) incorporates flat mirrors separated by a nominal distance of $1 \mathrm{~cm}$. This establishes a free spectral range of $v_{F S R}=15 \mathrm{GHz}$. The nominal finesse is $F \approx 150$ when the FPI entrance aperture diameter is less than $3 \mathrm{~mm}$. This decreases to about 30 when the full clear aperture of $12 \mathrm{~mm}$ is used. As explained below, we measure an effective finesse prior to a large number of similar measurements.

The FPI is scanned in frequency by movement of one of its mirrors, which is attached to three piezoelectric transducers. A ramp generator (EXFO RG-93) provides the necessary high voltage. With proper calibration by the user prior to experiments, the manufacture's design ensures that the mirror maintains its orientation parallel to the other mirror during translation

As indicated in figure 1, a tunable, external cavity diode laser (New Focus, Vortex 6000) is incorporated into the optical setup in such a way as to allow measurement of laser radiation instead of lamp radiation, without changing the basic optical layout. The laser is the one mentioned in section 1 that was used in the past for barium absorption measurements. In addition to general optical alignment, the laser is used to measure the system instrument function: the response of the FPI/optics to zero-spectral width radiation. This is necessary in order to properly extract from the data the spectral information of the barium emission. Details of this are discussed in section 3. In addition, the laser is used for comparisons between FPI emission measurements and laser absorption measurements of a barium hollow cathode lamp (see section 4).

\subsection{Electrical setup}

The electrical setup is presented in figure 2. A function generator provides a sinusoidal waveform to drive one of two amplifiers, depending on frequency of operation. For so-called low frequency operation $(500 \mathrm{~Hz})$, a California Instruments $251 \mathrm{~T}$ is employed, while for high frequency operation (10-100 kHz), an ENI 1140LA is employed. For the latter, a step-up transformer is required to boost the voltage and to ensure proper impedance matching with the lamp. In both cases, an ohmic ballast resistor, $\mathrm{R}_{\mathrm{V}}$, in the range of $200-500 \Omega$ is employed. Auxiliary coil heating current, $\mathrm{I}_{\mathrm{H}}$, can be supplied to the investigated electrode, using a floating d.c. power supply (Kikusui, PMC35-2A). 


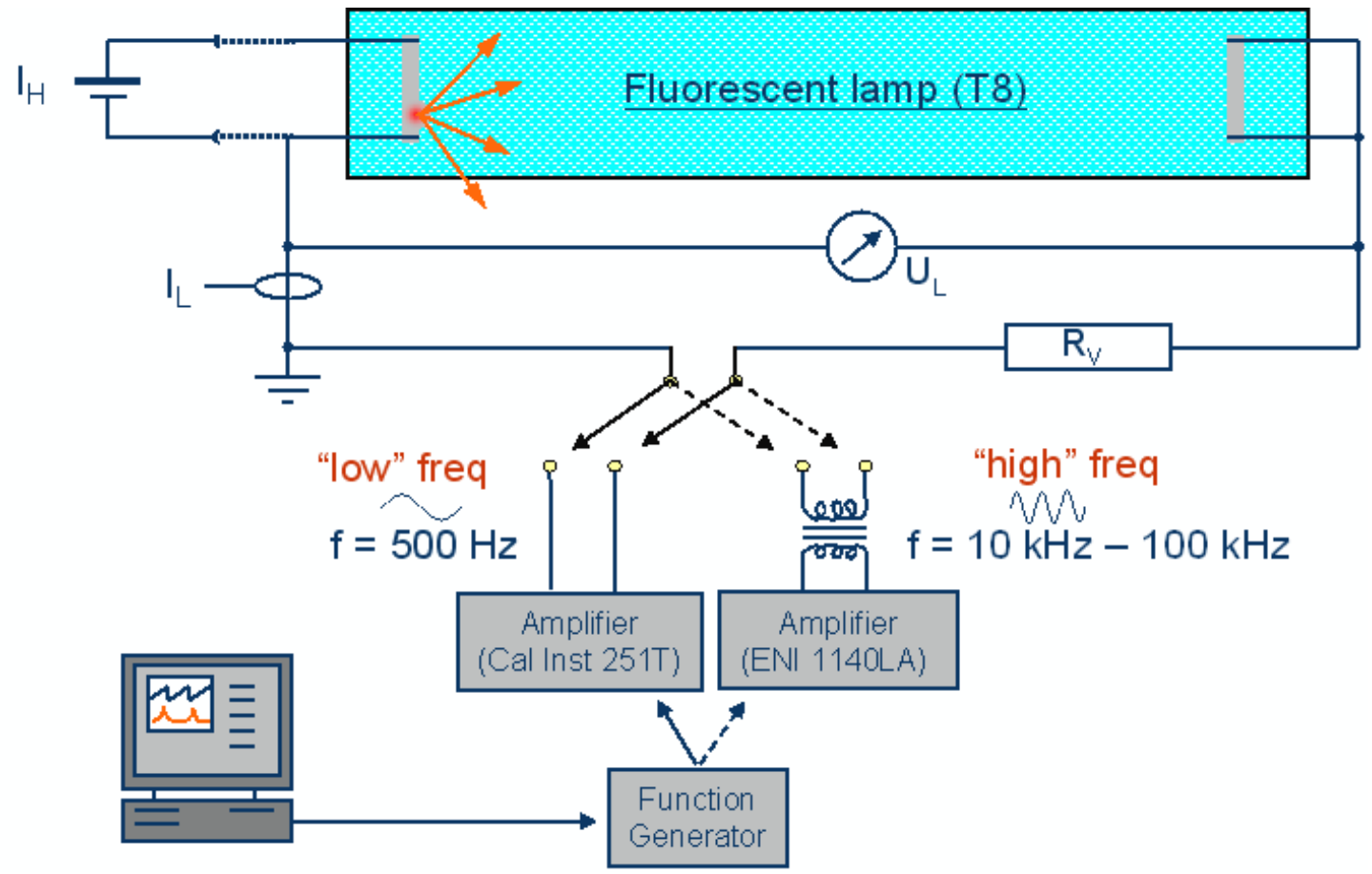

Figure 2. Electrical setup with lamp voltage $\mathrm{U}_{\mathrm{L}}$, discharge $\mathrm{I}_{\mathrm{L}}$, heat current $\mathrm{I}_{\mathrm{H}}$, and resistor $\mathrm{R}_{\mathrm{V}}$. A function generator drives one of two amplifiers, depending on low $(500 \mathrm{~Hz})$ or high $(10-100 \mathrm{kHz})$ frequency operation. In the latter case, a step-up transformer is necessary for voltage boosting and impedance matching.

For measurements on a.c.-driven lamps, the lamp current waveform and FPI voltage scan waveform are synchronized, thus allowing for measurement results that are phase-resolved with respect to the lamp current phase. The FPI ramp generator is externally driven so as to produce a sawtooth waveform with frequency of $1 \mathrm{~Hz}$. The external drive waveform is generated with a D/A converter in the computer using suitable software. Data is acquired during the rising portion, which represents $\sim 90 \%$ of the sawtooth. The falling portion is merely to reset the movable mirror to its starting position. During the rise, the voltage is held constant over each period of the lamp current waveform, and it is stepped by a discrete amount between successive periods. The spectral and phase information are untangled afterward in software.

Thus, for lamp measurements in which the current waveform period is $T$, there are $1 \mathrm{sec} / T$ steps of the FPI voltage for each $1 \mathrm{sec}$ sweep of the FPI. Typically, the FPI scan is over about two free spectral ranges $(30 \mathrm{GHz}$ ), making the frequency steps equivalent to approximately $30 \mathrm{GHz} \times T$. For example, for the low frequency lamp measurements $(500 \mathrm{~Hz})$, there are five hundred $60 \mathrm{MHz}$ steps. Data sample rate is typically $10^{6}$ samples/sec for both low and high frequency measurements, giving $1 \mu \mathrm{sec}$ temporal resolution within the waveform period. For improved signal-to-noise, the $1 \mathrm{sec}$ scan is repeated typically 300 times. In this scenario, data acquisition therefore takes approximately 5 minutes. 
The lamp voltage, $\mathrm{U}_{\mathrm{L}}$, is measured with a differential probe. The discharge current and auxiliary heat current are measured with a Hall current probe and a standard multimeter, respectively. The lamp voltage, discharge current, and PMT signals are recorded with an analog-to-digital (A/D) converter. Both D/A and A/D converters are located within the same data acquisition board (Data Translation, DT3010), inside a personal computer and controlled by a LabView program.

The investigated fluorescent lamps are similar to commercial T8 (1 inch diameter) lamps except that short sections of their ends have been cleared of phosphor to allow for transmission of barium emission out of the lamp tube. The lamps all have $110 \mathrm{~cm}$ length (electrode-to-electrode distance) and contain so-called stick (or double) coils of $12 \mathrm{~mm}$ length. The lamps contain either $100 \%$ argon $(\mathrm{p} \approx 1.3 \ldots 4$ mbar) or a mixture of $65 \%$ argon and $35 \%$ neon $(\mathrm{p} \approx 3.3 \mathrm{mbar})$. Some lamps contain mercury and some have just the buffer gas.

\subsection{Independent electrode temperature measurements}

Along with some of the FPI measurements, electrode temperature measurements are in addition made with a separate electrode temperature diagnostic (ETD) [10]. Results of some of these measurements are presented below and therefore we briefly discuss that diagnostic here. The ETD measurements provide support for the validity of the FPI diagnostic. The ETD employs a 128 pixel, InGaAs linear array detector (Hamamatsu, C7221) to sense the blackbody emission from the electrode in a $\sim 10 \mathrm{~nm}$ spectral window around $1.55 \mu \mathrm{m}$. The electrode, which is about $12 \mathrm{~mm}$ long and $0.8 \mathrm{~mm}$ in diameter, is imaged to the linear array with a magnification of $1 / 2$. Each pixel is $50 \mu \mathrm{m}$ in length $(\sim 6.4 \mathrm{~mm}$ total array length) and $200 \mu \mathrm{m}$ in width. Thus, the image of the electrode slightly underfills the linear array length, but overfills its width.

The viewing surface consists primarily of emitter material with possibly some of the tungsten coil windings showing in some places. In order to avoid the interpretational difficulties associated with the lack of knowledge of the thermal emissivity of the surface, a blackbody emission versus temperature calibration, for all points along the electrode, is obtained using the hot-to-cold resistance ratio technique. In this technique, the electrode is heated by passing current through it, with no discharge present. After the electrode has come to thermal equilibrium, the temperature, $T_{h}$, is calculated as follows:

$$
T_{h}=\rho^{-1}\left(\frac{R_{h}}{R_{c}} \rho\left(T_{c}\right)\right)
$$

where $R_{c}$ is the room temperature resistance, $T_{c}$ is the corresponding room temperature (both measured), and $\rho(T)$ is the known relationship between tungsten resistivity and temperature [11]. The function $\rho^{-1}(\rho)$ is the inverse of this relationship. Equation (1) assumes that the temperature is uniform, a reasonable assumption except within $\sim 1-2 \mathrm{~mm}$ from the posts which support the electrode (thus, the deduced temperature is perhaps a bit low). By performing this technique over a series of heating currents while measuring blackbody emission, the emission versus temperature is obtained. One then uses this to infer temperature from emission measurements while the discharge is on. Figure 3 shows an example set of measurements, elucidating the procedure. 


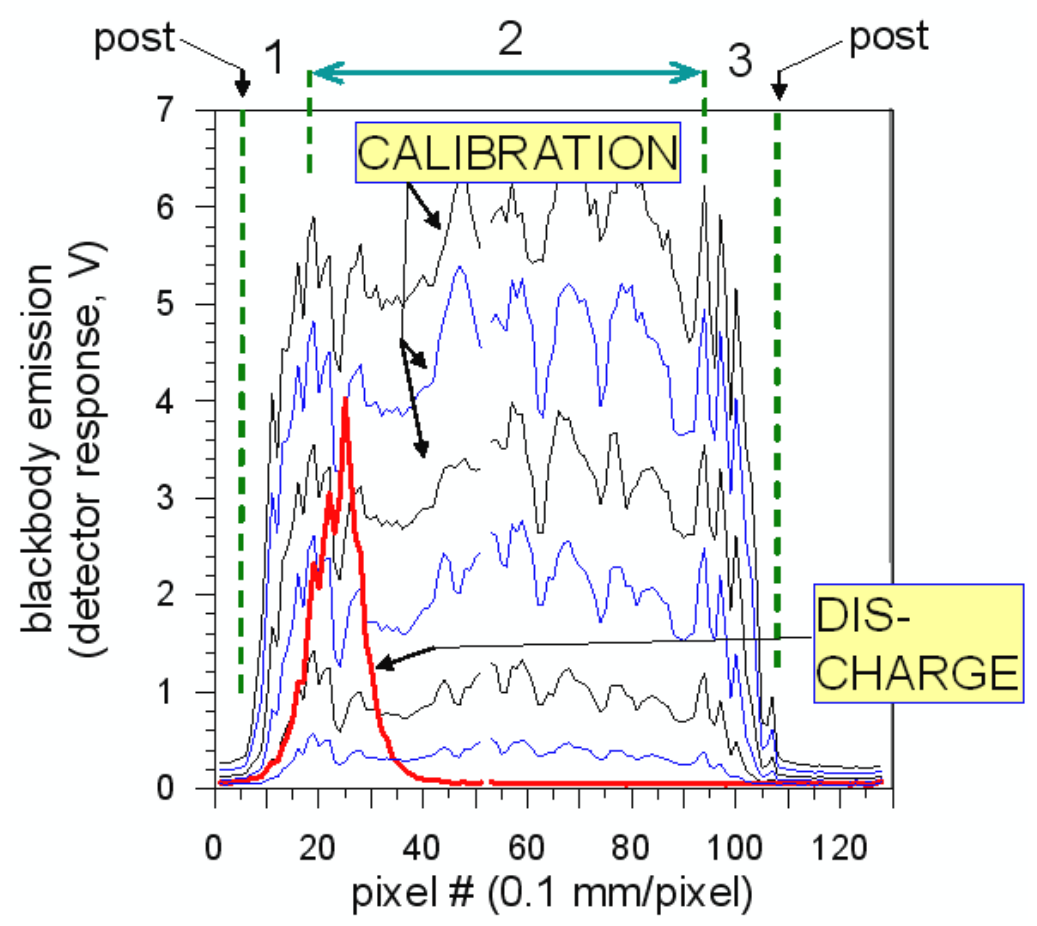

Figure 3. Demonstration of the electrode temperature diagnostic (ETD). The 'calibration' curves give the relative blackbody emission power (at $1.55 \mu \mathrm{m} \pm 5 \mathrm{~nm}$ ) for different applied coil currents. Each of these curves corresponds to a specific temperature, derived from the hot-to-cold resistance ratio. The procedure assumes uniform temperature during ohmic heating without a discharge, a reasonable assumption for the region labeled 2 in the figure. In regions 1 and 3 the temperature drops off quickly due to the high thermal conductivity of the posts that support the electrode. The 'discharge' curve gives the electrode's blackbody emission with the discharge on. Temperature at each pixel is deduced by interpolating the calibration curves. It is presumed that the spatial fluctuations are due to variations in emissivity, which exist due to surface irregularities.

\section{Data analysis and interpretation}

\subsection{System Response}

The system response function, $R\left(\Delta v_{F P I}\right)$, is the ratio of the sensed-to-emitted power spectral density as a function of the FPI frequency, $\Delta v_{F P I}$. The FPI frequency is established by the mirror spacing. The ' $\Delta$ ' is included to indicate that it is the change of the frequency (i.e., mirror spacing) from some arbitrary reference value that is important. $R\left(\Delta v_{F P I}\right)$ is a convolution of the instrument function, $I\left(\Delta v_{F P I}-\Delta v\right)$, with the spectral profile, $S(\Delta v)$, of the emitted radiation that is under investigation. The integration is with respect to the frequency of the radiation, $\Delta v$ (again, written with respect to a reference value, most conveniently the line center of the barium emission). It is recognized (see Appendix A) that the instrument function is a function of the difference between FPI and radiation frequencies. The instrument function equals the response function when $S(\Delta v)$ is the Dirac delta function. In practice, this is achieved when the source 
of radiation is the aforementioned diode laser, whose spectral width is $\sim 100 \mathrm{~s} \mathrm{kHz}$, much less than the free spectral range, $v_{F S R}=15 \mathrm{GHz}$, of the FPI.

In the present work, the spectral widths of the instrument function and of the barium emission radiation are of the same order (full-width-at-half-maximum (FWHM) $\sim 750 \mathrm{MHz}$ and $1 \mathrm{GHz}$ respectively), and it is necessary to untangle the two in order to determine the spectral width of the barium radiation alone. Therefore, it is important to consider the nature of the instrument function pertinent to the current work. The spectral width of the instrument function here is due to 1) the intrinsic spectral width of the FPI because of its finite finesse and 2) the apparent spectral width due to the angular spread of the radiation incident at the FPI. The two effects are of the same order and therefore the instrument function is significantly different from the well known, standard FPI instrument function for collimated radiation (which we refer to as the 'collimated instrument function').

An analytic form for the full instrument function, considering both effects, is derived in Appendix A. The full instrument function, $I\left(\Delta v_{F P I}-\Delta v\right)$, is given by equation (A.9), where $\mathrm{K}$ in that expression is the coefficient of finesse, which is related to the finesse, $F$, by equation (A.2). The quantity $F_{\theta}$ is an effective finesse associated with the angular spread and is given by equation (A.10). Equation (A.11) gives the collimated instrument function, $I_{\text {coll }}\left(\Delta v_{F P I}-\Delta v\right)$, an Airy function in its argument, appropriate when the incident radiation is incident at zero angle. When the radiation frequency spans a range much less than $v_{F S R}$, then the collimated instrument function can be approximated by a Lorentzian, displayed in equation (A.12). It is shown in Appendix A that the Lorentzian approximation of the collimated instrument function is suitable for the current work, in spite of the non-zero angular spread of the incident radiation. Finally, Equation (A.7) is the response function, where one can insert the desired instrument function.

\subsection{Instrument function measurement}

Prior to a large set of similar measurements of barium emission from the lamp, the diode laser is used to measure the instrument function. The measurement is essentially identical in every respect to the lamp measurement except that laser radiation is directed through the optical system. The instrument function, as measured, includes the effect of the angular spread that is established by the pinhole and lens L2. This is made clear in figure 4, which shows a subset of the optical system along with various parameters of the laser beam as it propagates through. It is assumed that the beam from the laser has a fundamental, cylindrically symmetric Gaussian mode shape, and therefore the standard formulae for propagation of such beams are used [12].

As indicated in the figure, lens L1 images the waist of the laser output beam to approximately the focal plane of lens L1, located midway between L1 and the pinhole A1. The radiation between $\mathrm{L} 1$ and $\mathrm{A} 1 \mathrm{has}$ a Rayleigh range of $1.7 \mathrm{~mm}$ and therefore $\mathrm{A} 1$ is in the far field with respect to the waist in this region. The phase fronts of the radiation at the plane of pinhole are thus approximately spherical and have $\sim 1 \mathrm{~mm}$ diameter at this point, significantly overfilling the 100 $\mu m$ diameter pinhole. The spherical phase fronts just within the opening of the pinhole are nearly flat (full angle spread of $\sim 10^{-4} \mathrm{rad}$ ) and with nearly constant intensity (edge/peak intensity 0.9). In this case, the intensity versus angle at lens L2 approaches that of the standard Fraunhoffer diffraction pattern that results from plane waves incident upon a circular aperture [13]. The full angle spread is $\theta_{\text {diff }}=1.22 \lambda / d_{\text {pin }} \approx 8 \mathrm{mrad}$. This radiation is converted by lens 
L2 to radiation with approximately spherical phase fronts at the FPI which have (full) angular spread of $3.33 \mathrm{mrad}$ (twice the maximum angle of incidence at the FPI cited above).

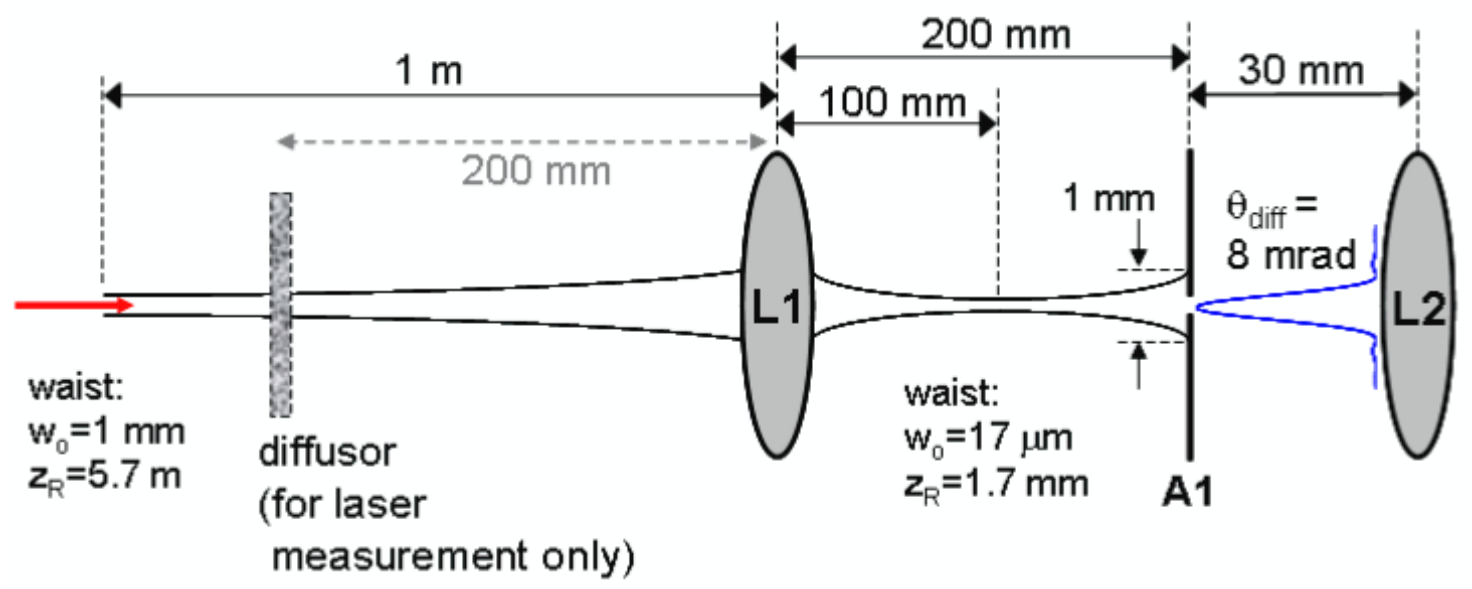

Figure 4. Subset of the optical system (shown in Figure 2), highlighting detail of laser beam propagation through the optics prior to its incidence at the FPI. The laser is used for measurements of the instrument function, the response of the system to zero spectral width radiation. The figure demonstrates that the use of the laser beam leads to the same angular spread induced spectral width. Thus, a proper instrument function is determined, in the sense that the laser beam and barium radiation differ, at the FPI entrance aperture, only in their spectral content. This is validated by employing the diffuser with the laser measurement, which produces the same spectral response (except for overall diminished signal) as with the diffuser absent.

Support for this theoretical argument is provided by comparing measurements of the laser radiation both with and without a diffuser placed in the optical path. The diffuser is placed at a location $200 \mathrm{~mm}$ in front of lens L1, and therefore the diffuse laser spot thus produced is directly imaged to the pinhole. The spectral profiles, determined with the FPI, of laser radiation both with and without diffuser are the same, and have FWHM of about $750 \mathrm{MHz}$. Furthermore, the spectral profile of the laser radiation measured by sending the laser beam directly into the FPI (no intermediate optics) is narrower, with FWHM of approximately $500 \mathrm{MHz}$.

\subsection{Data reduction}

After appropriate rearrangement, as discussed in Section 2.2, the raw data consists of a set of response functions, $R_{\delta t}\left(\Delta v_{F P I}\right)$, as a function of $\Delta v_{F P I}$ at different times, $\delta t$, during the period of the lamp current waveform. The ' $\delta$ ' indicates that these are really small time ranges equal to the inverse of the sample rate $\left(10^{6} \mathrm{samples} / \mathrm{sec}\right)$. The desired information is the spectral width, at each time, of the barium emission radiation, which is characterized by source function $S_{\delta t}(\Delta v)$. The task is to extract this from the data using the representative model of equation (A.7).

Our approach is to perform a nonlinear least squares fit of (A.7) to the data, with the FWHM (or equivalent figure-of-merit (FOM)) of the source function as a free parameter, and using the 
coefficient of finesse derived from the separately-measured instrument function. In order to do this, a functional form for the source function is required, with the FOM as a free parameter. For this, we choose a Gaussian, and the FOM is the standard deviation. This is the natural choice for evaporated barium, but not necessarily for sputtered barium. We address this issue in the next subsection (3.4).

The instrument function is measured with the laser in the manner described in section 3.2. The coefficient of finesse, $\mathrm{K}$, is obtained by a least squares fit of the instrument function to these data. One approach is to use the full instrument function (A.9) for these purposes, with $\mathrm{K}$ as a free parameter, and using the known value of $\theta_{m}=1.67 \mathrm{mrad}$. It is found that this provides, in the end, a similar spectral width for the source function, if instead the Lorentzian approximation to the collimated instrument function (A.12) is used. One then derives an erroneously larger intrinsic finesse since it has the angular spread finesse incorporated into it. This nonetheless produces a similar source function width because the main difference between full and collimated instrument functions (either the "true" or the Lorentzian approximation) are in the wings far from the peak, where instrument functions and data have small values. Justification of this approach is provided in Appendix A. In any case, the small errors in the absolute value of the derived source function widths are not consequential here, since we are mainly interested in sensing the differences in temperatures between evaporated and sputtered barium.

\subsection{Source function considerations}

It is beyond the scope here to delve deeply into the theoretical framework underlying the velocity distribution function that is at the heart of the measurements, since this involves a complicated interplay of a multitude of mechanisms going on in the discharge. The main intent of the present work is to differentiate between sputtered and evaporated barium, if indeed the former occurs at all. To the extent that this has been successful is supported by the measurements themselves. Nonetheless, it is important to be aware of some basic aspects of the framework to better understand and interpret the measurements.

Atomic line emission that is detected from the low pressure discharge environment of a fluorescent lamp is Doppler broadened. Broadening due to collisions is negligible ${ }^{\S}$. Therefore, the frequency spectrum has a profile indicative of the distribution of the components of the velocities, $\mathrm{v}_{t}$, along the line of site between atomic emitters and observation point. If the distribution is given as $f\left(\mathrm{v}_{t}\right)$, then the frequency spectrum is $S(\Delta v)=f(\lambda \Delta v)$, where $\Delta v$ is the frequency with respect to the natural line center of the radiation and $\lambda$ is the wavelength.**

In the present work the emission that is sensed comes from atomic radiators distributed along a path within the lamp. Therefore, we must be mindful of the fact that we actually sense some weighted integral of the distribution function along this path. This complication is compounded by the fact that the emission is due to the number of atoms in the excited state of interest which,

\footnotetext{
$\S^{\S}$ Collisional broadening associated with the time between collisions (assuming zero interaction time) is estimated to have at most 10's MHz characteristic frequencies, compared to the 100's MHz to $\sim 1 \mathrm{GHz}$ Doppler broadening frequencies. Broadening resulting from non-zero interaction times (in particular van der Waals broadening due to rare gas atoms) is expected to be negligible, a notion somewhat justified by observations in this work: such broadening would induce asymmetries in the spectrum, which are not seen. ** Henceforth, we refer to $f\left(\mathrm{v}_{t}\right)$ as the velocity distribution function with the understanding that it is the distribution of one component of the velocity, independent of the values of the two mutually orthogonal components.
} 
in turn, is due to the plasma parameters (electron density and temperature, both a function of time and position) that lead to their excitation. The measurement time ( $\sim 1 \mu \mathrm{sec})$ can be considered short enough such that the distribution function at each point in space does not change during that time.

The functional form of distribution function at a particular time and position is related to its form just outside the hot spot at the time of the escape of the barium (the 'source distribution'), and to the subsequent interactions to which the barium is subject subsequent to that escape. The latter collectively constitutes the barium transport problem, a key determining factor of the true rate of loss of barium from the electrode. The source distribution is essentially a boundary condition for the set of differential equations which describe the transport problem.

In the ideal sense of the current work, we simply sense the differences in the source distributions that are a consequence of the differences between the evaporative and sputtering mechanisms. For the case of (neutral) barium atoms that are evaporated from the hot spot, standard thermodynamic arguments suggest [14] that the source distribution is Maxwellian with temperature equal to that of the hot spot, $T_{K}$. Thus, in the ideal sense, the spectral profile of the barium emission would have a Gaussian functional form with a standard deviation, $\sigma_{\text {evap }}$, given as:

$$
\sigma_{\text {evap }}=v_{o} \sqrt{\frac{k_{B} T_{K}}{m c^{2}}}
$$

where $\mathrm{m}$ is the mass of barium, $\mathrm{c}$ the speed of light, $k_{B}$ the Boltzmann constant, and $v_{o}$ the frequency of the line center.

In the case of sputtered atoms the source distribution is not as well understood, especially for the low energy type of sputtering that characterizes the present situation. One type of distribution which has been used to characterize sputtered particles from elemental targets is the 'cascade distribution' which, written in terms of the energy, E, of the particles, and normalized to unity, is [15]

$$
f_{\text {cascade }}(E)=2 \frac{E U}{(U+E)^{3}}
$$

where $U$ refers roughly to a surface binding energy. The distribution apparently does well for incident ion energies $>1 \mathrm{keV}$, and there is evidence that it is suitable for $<1 \mathrm{keV}$, with perhaps modifications of the value of the power to which the denominator is raised.

It is straightforward to transform (3) into a distribution for a component of the velocity along one dimension (assuming isotropy), given that the mutually orthogonal components can take on any values. This is described in Appendix B, with the result given in Equation (B.6). The function is graphically represented in Figure B1 and shows the cascade velocity distribution to have shape similar to that of a Gaussian, but with an extended tail (and infinite standard deviation). If a Gaussian function is fit, in least squares sense, to the cascade velocity distribution, with the standard deviation and amplitude as free parameters, then the standard deviation of that Gaussian corresponds to an energy which is about 7\% less than the speed associated with the binding energy (i.e., $\left.\sigma_{\mathrm{v}} \sim 0.933 \times(2 U / \mathrm{m})^{1 / 2}\right)$. Therefore, in the present work, if we simply assume a 
Gaussian shape for the sputtered atoms, then we should expect an effective temperature that is approximately equal to the binding energy (in the ideal sense, not considering interactions subsequent to the sputtering, which are discussed below).

The binding energy in this scenario is not precisely defined, but it is believed to be on the order of the heat of sublimation [15]. For the present situation of barium atoms at the surface of the triple oxide emitter, this has been measured and is approximately equal $75 \mathrm{kcal} / \mathrm{mole}=3.26$ $\mathrm{eV} /$ particle. ${ }^{\dagger \dagger}$ Even if this is not precisely correct, it is likely that the binding energy is significantly greater than typical measured values of $k_{B} T_{K}(\sim 0.11 \mathrm{eV})$. Thus, we should expect that sputtered atoms have a much higher effective temperature than that of evaporated barium. Of course, the temperature associated with the above estimated binding energy is quite high $\left(\mathrm{U} / \mathrm{k}_{\mathrm{B}} \sim 37800^{\circ} \mathrm{K}\right)$ and we do not expect to see (indeed, have not seen) such effective temperatures.

It is presumed that collisions with the very plentiful rare gas atoms reduce the temperature significantly. We estimate an energy relaxation rate of approximately $\varepsilon_{E}>3.5 \times 10^{6} \sec ^{-1 \mathrm{t}}$, where the lower bound corresponds to a barium atom energy of $0.1 \mathrm{eV}$ and higher values corresponds to proportionately higher energies. This is based on a rough calculation, but it is intended to show that the energy relaxation to the background gas temperature can be quite quick. Therefore, the fact that we observe temperatures beyond thermal for the sputtered barium (in the present experiments and the previous laser absorption measurements) presumably indicates residual energy left over from the sputter mechanism which imparts much greater energy. Of course, to be more rigorous, one must take into account the spatio-temporal transport of particles and energy.

\section{Laser absorption measurements}

The laser absorption measurements of the previous work [9] provide support for the validity of the present measurements since they exhibit a more direct and unequivocal demonstration of the high effective temperatures of sputtered barium $\left(>2000^{\circ} \mathrm{K}\right.$ for situations in which the cathode is near room temperature). Therefore, it is fitting that we provide a brief description of, and some results from, that work. The setup is straightforward. The diode laser beam is directed through the lamp near the electrode, then passes through an interference filter, and is subsequently sensed by a silicon PIN photodiode. The diode's output is directed to a current amplifier. The laser frequency is scanned over the line of interest with a sawtooth waveform. The scan (sawtooth) period for the results shown below is $1 \mathrm{msec}$, and the spectral frequency range of the scan is

\footnotetext{
$\dagger$ This has been measured by the optical absorption technique for the situation in which barium is evaporated from an electrode that is heated by application of current, without a discharge [4]. Using a barium hollow cathode lamp as a radiation source, the transmission of $553 \mathrm{~nm}$ radiation through the evaporated barium as a function of applied current is determined. Average barium density, $\mathrm{n}_{\mathrm{Ba}}$, is deduced assuming a Beer-Lambert law for transmission. Electrode temperature, $\mathrm{T}_{\mathrm{K}}$, is deduced from the hot-to-cold resistance ratio. Heat of sublimation, $\alpha$, is then obtained from a linear fit of $\ln \left(n_{B a} T_{K}\right)$ versus $1 / T_{K}$, which has slope $-\alpha[14]$.

\# Calculated by considering $\mathcal{E}_{E}=v_{\text {coll }} \xi$, where collision frequency $v_{\text {coll }}=n_{\text {gas }} \sigma_{\text {coll }} \mathrm{v}_{\text {rel }}$ and $\xi=2 m_{g a s} m_{b a} /\left(m_{g a s}+m_{b a}\right)^{2}$ is roughly the fraction of kinetic energy transferred per collision [16]. Elastic collision cross section $\sigma_{\text {coll }} \approx 5.72 \times 10^{-14} \mathrm{~cm}^{2}$, relative velocity, $\mathrm{v}_{\text {rel }}$, is taken to be that associated with a barium atom with $0.1 \mathrm{eV}$ of kinetic energy, and $\mathrm{n}_{\text {gas }}$ is the density of argon at 2.5 Torr pressure and $1000^{\circ} \mathrm{K}$ temperature.
} 
about $16 \mathrm{GHz}$, enough to encompass the main line plus some hyperfine and/or isotope shifted lines as well.

Figure 5 shows the results of one scan a few milliseconds after initial breakdown of a T8 fluorescent lamp. The lamp was operated in a manner similar to the "low frequency" mode of operation described in section 2 (see figure 2) except that the driving waveform was $60 \mathrm{~Hz}$. The lamp contained argon buffer gas at roughly 2.5 Torr.

The figure shows a plot of optical depth (absorption coefficient $\times$ path length) versus laser frequency (arbitrary zero point). Four lines are observed, which reveals the hyperfine and isotope shift structure of the transition of interest. The most prominent line in figure 5 corresponds to the transition of interest in ${ }^{138} \mathrm{Ba}$, which is the most abundant isotope $\left(71.7 \%\right.$ [17]). ${ }^{138} \mathrm{Ba}$ has zero nuclear spin and therefore the atomic transition has no hyperfine structure. The other three lines correspond to isotope shift, most likely of ${ }^{135} \mathrm{Ba},{ }^{136} \mathrm{Ba}$, or ${ }^{137} \mathrm{Ba}$, the next most abundant isotopes $(6.6 \%, 7.8 \%, 11.3 \%$ respectively [17]) and/or hyperfine structure of the barium isotopes with odd mass, which have non-zero nuclear spin. The precise association of the lines can only be ascertained with additional measurements that were not within the scope of the work.

The effective temperature of the barium (derived from the most prominent line, although the other lines give similar results), is $2140^{\circ} \mathrm{K}$. This is much greater than the presumed temperature of the electrode, which is only slightly above room temperature during this point in the starting phase. In any case, the final hot spot temperature during thermionic steady-state is only about $1400^{\circ} \mathrm{K}$. It is noted that the high temperature persists throughout the starting phase and, in fact, decreases, when the electrode goes thermionic (measurable only occasionally when high barium densities persist for a little while after the starting phase ends). Thus, it is plausible that high effective temperatures also observed during steady-state through emission measurements utilizing the FPI, as we shall see in the next section, are also due to sputtered barium.

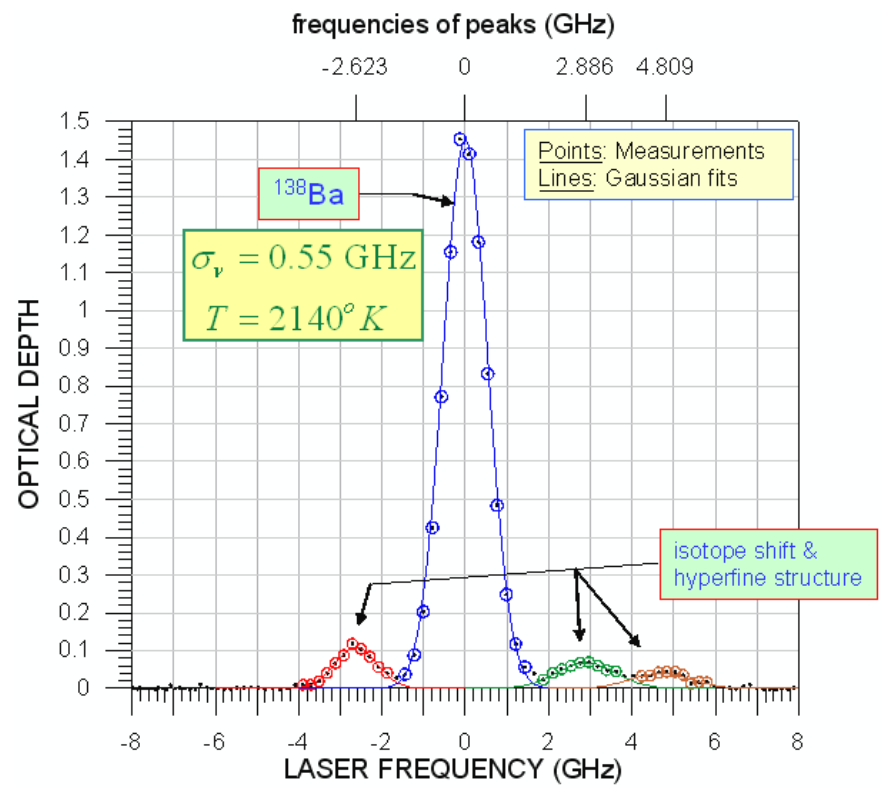


Figure 5. Optical depth versus frequency derived from laser absorption measurements on the barium transition $(6 s 5 d)^{3} D_{3} \rightarrow\left(5 d 6 p^{\prime}\right)^{3} D_{3}^{0}$ (center wavelength: $649.8762 \mathrm{~nm}$ in vaccum). The measurement is during a $1 / 2$ msec time period after the initial breakdown of a T8 Lamp with 2.5 Torr argon buffer gas. The frequency scale is zeroed at the center of the most prominent line, which corresponds to the transition in ${ }^{138} \mathrm{Ba}$, the most abundant isotope. Other lines observed are some combination of isotope shift and hyperfine structure of the barium isotopes with odd atomic number. The precise identification requires additional measurements which are outside the scope of the current work. The barium (effective) temperature, derived from the most prominent line, is $2140^{\circ} \mathrm{K}$, much greater than the electrode's temperature, which is presumed to be just a little greater than room temperature.

\section{Fabry-Perot Measurements}

\subsection{Hollow Cathode lamp measurements}

To verify the applicability of the Fabry-Perot interferometer technique, preliminary investigations were performed on a barium hollow cathode lamp (HCL). By design, an HCL lamp operates with high cathode falls in order that the atoms of interest are sputtered. The temperature of barium atoms sputtered from the electrode of a barium HCL was measured with both the FPI and the laser absorption technique. Laser absorption measurements were performed in a manner similar to that described in section 4. For the FPI measurements, the HCL replaced the fluorescent lamp in the arrangement shown in figure 1. Measurements were made of the emission corresponding to the same transition as that measured by laser absorption.

Figure 6 shows the result of the laser absorption measurement in a plot of absorbance $\left(\log _{10}\left(\mathrm{I} / \mathrm{I}_{0}\right)\right)$ versus laser frequency. A Gaussian fit of the data gives a FWHM of $1.05 \mathrm{GHz}$, which corresponds to an effective temperature of $1388^{\circ} \mathrm{K}$. The frequency scan covered only the line of the most abundant isotope ${ }^{138} \mathrm{Ba}$.

Figure 7 shows the emission measurement in a plot of power versus FPI frequency. The upper plot shows a measurement of the instrument function made with the laser in the manner described in section 3. Fitting the collimated instrument function to these data, the FWHM is $780 \mathrm{MHz}$. The lower plot shows the emission from the HCL. Deconvolving this and instrument function measurements, in the manner described in section 3 (utilizing the measured coefficient of finesse) gives an effective barium temperature of $1380^{\circ} \mathrm{K}$, nearly the same as the laser absorption measurement.

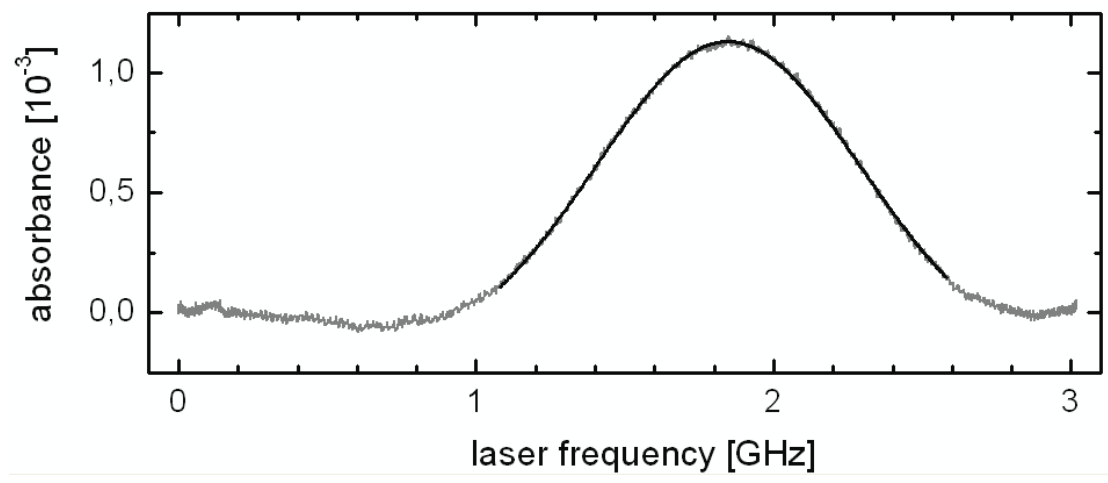


Figure 6. Laser absorption measurement of barium in a steady-state barium hollow cathode lamp using the diode laser at $\sim 650 \mathrm{~nm}$. In comparison to the results shown in figure 5 for a fluorescent lamp, the scan over frequency covered only the most abundant isotope, ${ }^{138} \mathrm{Ba}$. A Gaussian fit gives a temperature of $1388 \mathrm{~K}$.

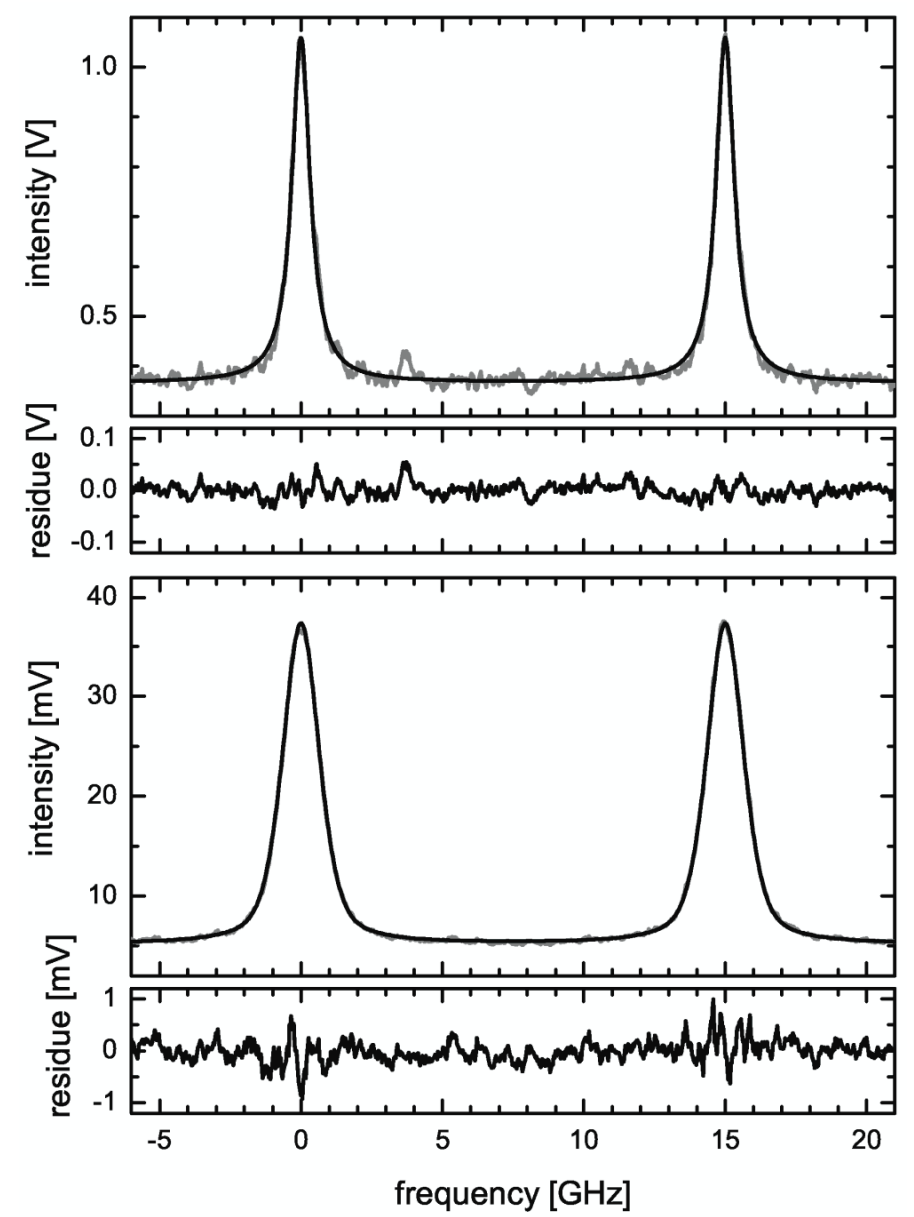

Figure 7. FPI instrument function measurement at $650 \mathrm{~nm}$ using the diode laser (upper). Emission measurement $(650 \mathrm{~nm})$ from a steady-state barium hollow cathode lamp (lower). Deconvolving in the manner described in the text gives a temperature of $1380 \mathrm{~K}$, similar to that derived from the laser absorption measurement shown in figure 6 .

\subsection{Fluorescent lamp measurements}

Results are shown here of measurements on fluorescent lamps. The investigation has shown that mercury-free, rare gas discharge lamps exhibit sputtering over a broad parameter range. Therefore, many of the results shown will be for such lamps in order to show the essential features of sputtering behavior. Mercury-containing lamps in general do not exhibit sputtering unless operated in unusual parameter regimes. This is consistent with our expectation that rare gas discharges have higher cathode falls than mercury-rare gas discharges because the ionization potentials of the rare gases are higher than that of mercury. 
Figure 8, 9 and 11-15 show results of measurements performed on a mercury-free $100 \%$ argon discharge with pressure 2.5 Torr, whereas figure 10 shows measurements performed on a mercury-argon discharge with the same pressure. Eight measurements performed under identical conditions are shown in figure 8 , indicating the reproducibility of the results. The lamp was grounded at one of the posts of the investigated electrode, as depicted in Figure 2. A hot spot temperature of $1500^{\circ} \mathrm{K}$ was measured using the electrode temperature diagnostic (section 2.3). The figure shows the FPI-derived barium temperature (upper), the intensity of the barium emission (middle), and the lamp voltage and current (lower) over one cycle of a lamp operating with $270 \mathrm{~mA}$ rms discharge current at $500 \mathrm{~Hz}$. In the intensity and temperature plots, the solidline portions of the curves refer to uncertainties of less than $10 \%$ in the least-squares fit used to extract the information from the measurement (as described in Section 3.3). The dashed-line portions refer to uncertainties of between $10 \%$ and 33\%. Those portions of curves with uncertainties greater than $33 \%$ are left blank. The current is plotted inverted for clarity.

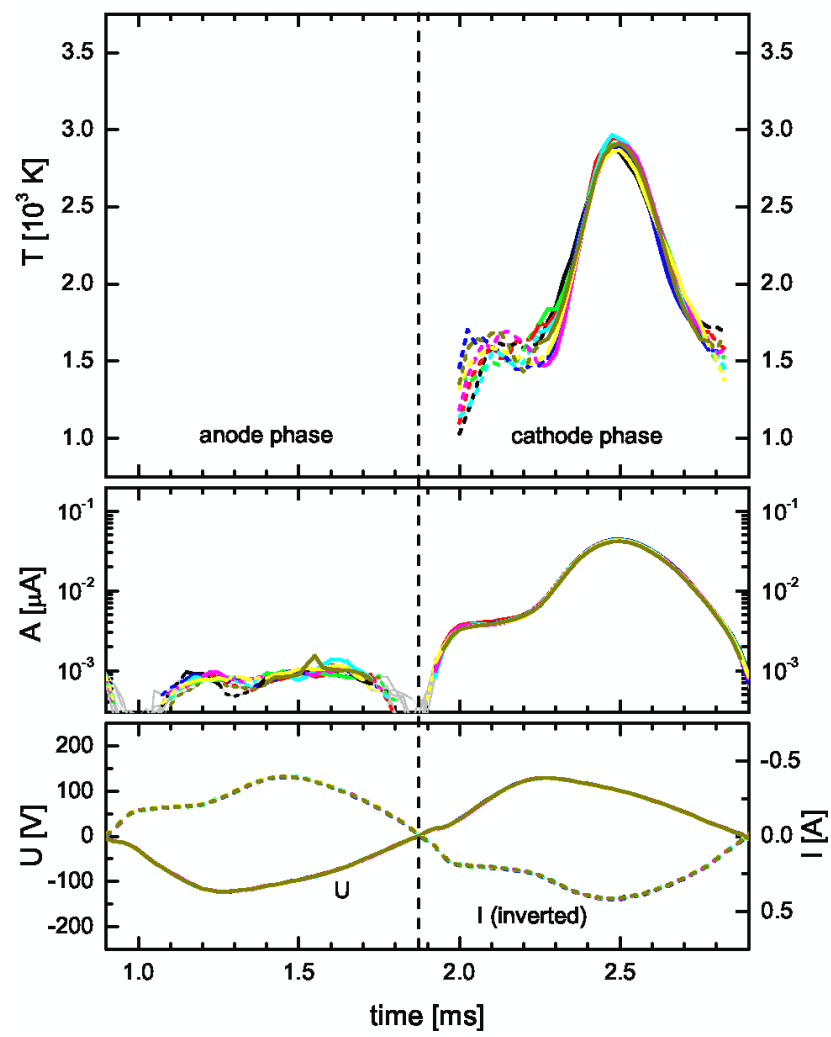

Figure 8. Barium temperature (above), intensity of the barium signal (middle) and voltage/current (below) of a mercury free argon discharge lamp ( $p=2.5$ Torr) at $270 \mathrm{~mA}$ for eight identical measurements (line colors). In this and succeeding figures, the solid and dashed lines represent data with an error less than $10 \%$ and between 10 and 33\%, respectively. Data with an error above 33\% are not shown. The current is plotted inverted for clarity. Barium is presumed to be sputtered when the temperature increases above the hot spot temperature, which is about $1500^{\circ} \mathrm{K}$.

As seen in the figure, the barium temperature can be determined accurately only during the cathode phase, when the barium emission intensity is relatively high. Intensity can be determined 
during the anode and cathode phases, but it becomes immeasurably small around the times of the zero-crossings of the current.

In the cathode phase, there are two distinct periods during which the intensity and temperature have different behavior. There is a $\sim 200 \mu \mathrm{sec}$ period near the beginning of the cathode phase during which the intensity and temperature are slowly changing. Temperature at this time is about $1500^{\circ} \mathrm{K}$, similar to the independently-measured hot spot temperature. We conclude that barium evaporates during this time. Prior to this initial period, the intensity and temperature increase rapidly, although the large uncertainties in temperature may render this interpretation questionable.

Following this initial period, the intensity and temperature increase quickly, reach a peak at the time of the current maximum, and then decrease, similar to the behavior of the current. Maximum barium temperature is $\sim 3000^{\circ} \mathrm{K}$, much higher than the hot spot temperature. Hot spot temperature is fairly constant due to the relatively large thermal mass of the electrode. We conclude that barium is sputtered during this time. The high (effective) temperatures are remnants of the even higher energies induced by the sputtering event, as discussed before (section 3.4).

The notion that barium sputters, not immediately but after some time in the cathode phase, is consistent with our understanding of the phase dependence of the cathode fall [5]. When the electrode first enters the cathode phase, the discharge current is relatively low. Meanwhile the electrode is fairly hot. Its large thermal mass precludes its temperature from varying very much over phase, even at the relatively low $500 \mathrm{~Hz}$ operation. Thus, the electrode is able to supply the needed electron flux thermionically during the early part of cathode phase. However, as discharge current increases further into the cathode phase, the additional electron flux required cannot be supplied through increases in electrode temperature. The dearth of electrons causes the cathode fall to increase, leading to increased electric field at the surface of the electrode and increased field-assisted thermionic emission (Schottky and patch effects).

Figure 9 shows a similar set of plots as figure 8, but now for a series of currents in the range of $190 \mathrm{~mA}$ to $470 \mathrm{~mA} \mathrm{rms}$. It is notable that sputtering occurs over this entire range, fairly broad with regard to fluorescent lamp operation. The general trend just described is the same but with slight variations. The peak temperatures decrease and the intensities increase with increasing rms current. The precise times of the peaks shift in phase relative to the peak of the current during the cathode half cycle. For the two highest rms currents of $440 \mathrm{~mA}$ and $470 \mathrm{~mA}$, temperatures in the anode phase could be determined and their levels suggest that barium is evaporated during that time.

Figure 10 shows similar results, but for a mercury-argon discharge with about the same pressure. In contrast to the mercury-free discharge, sputtering occurs only at the relatively low current of $60 \mathrm{~mA}$. It is presumed that sputtering would occur at even lower currents, but an extensive investigation of this has not been carried out. This is consistent with the fact that cathode fall increases as discharge current decreases [5]. It is noted that when barium exhibits only evaporation, the emission intensity is almost constant in time.

Figure 11 shows the effects of the application of auxiliary coil heat current on the mercury-free discharge. Results are shown for a series of d.c. heat currents from zero to $500 \mathrm{~mA}$. The discharge currents are not identical but vary only slightly in the range of $240 \mathrm{~mA}$ to $280 \mathrm{~mA} \mathrm{rms}$. With increasing heat current the temperature (especially at the peak) decreases. For an auxiliary 
coil heat of $500 \mathrm{~mA}$ no barium is sputtered. This is consistent with the fact that cathode fall decreases with increasing heating current [5].

Figure 12 shows a compendium of the coil heat results. Shown are plots of peak temperature (upper) and cathode phase-integrated intensity (lower) versus coil heat current. It is seen that peak temperature decreases approximately linearly with increasing heat current. The integrated intensity at first decreases and then increases with heat current, with a minimum somewhere in the $300 \mathrm{~mA}$ to $400 \mathrm{~mA}$ range. The latter behavior correlates well with LIF measurement performed on similar T8 lamps in previous work [18], except that the minimum barium density in front of the electrode was found to be in the range of 400 to $500 \mathrm{~mA}$ heat current. The LIF lamps in that work were driven with a higher discharge currents $(500 \mathrm{~mA})$, more typical of those in Europe.

The next result shows the effect of the variation of the location of the hot spot on one particular electrode of a mercury-free lamp. A fortuitous change in hot spot position, coupled with a difference in emitter properties at these two positions (in this case, presumably, work function) gives rise to this rather interesting result which serves to strengthen our confidence in the efficacy of the diagnostic. Figure 13 shows pictures of two different hot spots which existed at different times, both near the posts at opposite sides of the same electrode. Visually, the two hot spot are obviously different. The upper picture ("post 1") shows an evidently cooler hot spot which has a larger spatial extent, while the lower picture ("post 2") shows a hotter, shorter hot spot. The electrode temperature diagnostic provides peak temperatures of $\sim 1200^{\circ} \mathrm{K}$ and $\sim 1500^{\circ} \mathrm{K}$ respectively.

Figure 14 shows the results of FPI measurements of these two hot spots, presented in the same manner as before. It is seen that the hotter hot spot exhibits sputtering in similar fashion to the mercury-free results shown above. The cooler hot spot does not exhibit sputtering. Furthermore, the FPI-derived temperatures during the evaporative regimes of both hot spots agree well with the emission-based diagnostic. This result agrees well with our notion of how electrodes work [5]. The emitter at post 1 presumably has lower work function than the emitter at post 2 . This allows it to thermionically emit similar fluxes of electrons at lower temperature. This is accompanied by a lower cathode fall since maintaining the lower temperature requires lower ion bombardment energy. The lower cathode fall leads to much less (or possibly no) sputtering.

Figure 15 shows the behavior at high operating frequencies. Shown are plots of temperature, intensity, voltage, and discharge current versus phase degree during the course of one cycle. Each curve corresponds to a different frequency in the range of $10 \mathrm{kHz}$ to $100 \mathrm{kHz}$. The $500 \mathrm{~Hz}$ case shown before is included here for easy comparison. It is apparent that the general trend seen for the $500 \mathrm{~Hz}$ case becomes more smeared out as frequency is increased. Barium temperature exhibits a tendency to increase during the cathode phase, but it does not decrease, either in the late cathode phase or anode phase, to the amount what would be considered evaporative levels. The variations in barium temperature with phase are smaller as frequency is increased. At 100 $\mathrm{kHz}$, the temperature and intensity are almost constant throughout the cycle. For all high frequency cases, barium temperature is quite high during the entire phase. It averages about 2300 $\mathrm{K}$, well above the hot spot temperature. This does not suggest that sputtering occurs during the entire cycle since one has to take into account the full particle and energy transport problem. It merely suggests that sputtering occurs at some point in the cycle.

Figure 16 shows a compendium of results for various pressures. Discharge currents were not the same, but all were in the range of 200-300 mA. Operating frequency was $500 \mathrm{~Hz}$. Both mercury- 
free (red) and mercury-containing lamps (black) are depicted. Four of the lamps contained a mixture of $65 \%$ argon and 35\% neon (two mercury-free and two with mercury), while the rest were all $100 \%$ argon. The results for 2.5 Torr overlap the results shown in previous figures. The upper plot shows the maximum barium temperature during the cycle while the lower plot shows phase-integrated barium emission intensity.

Some notable characteristics of these data are as follows. The mercury-containing lamp with 1 Torr argon pressure exhibits sputtering (in contrast to the 2.5 Torr mercury-containing lamp, as we saw before). This is consistent with the fact that cathode fall increases as pressure decreases [2]. Furthermore, it is well known that T8 lamps with such low pressure experience severe end darkening and significantly shortened life. The mercury-containing lamp with 65\% argon and $35 \%$ neon also exhibits sputtering, although apparently not as severe as the 1 Torr lamp. This also is consistent with the increased cathode fall such lamps have, relative to their $100 \%$ argon counterparts with the same total pressure.

The mercury-free lamps exhibit sputtering across the pressure range depicted. Since mercuryfree lamps exhibit sputtering at 2.5 Torr, then one would expect them to also exhibit sputtering at lower pressure, where cathode falls are higher. The mercury-free lamp with some argon replaced by an equal amount of neon exhibits sputtering. This is consistent also with higher cathode falls for such lamps ${ }^{\S \S}$.

\footnotetext{
${ }^{\S}$ Higher ion mobility in neon compared to argon leads to faster ambipolar diffusion, faster charged particle loss rates, and therefore the need for higher ionization rates by beam electrons. This is accomplished through higher cathode fall, which imparts greater energy to the beam electrons.
} 


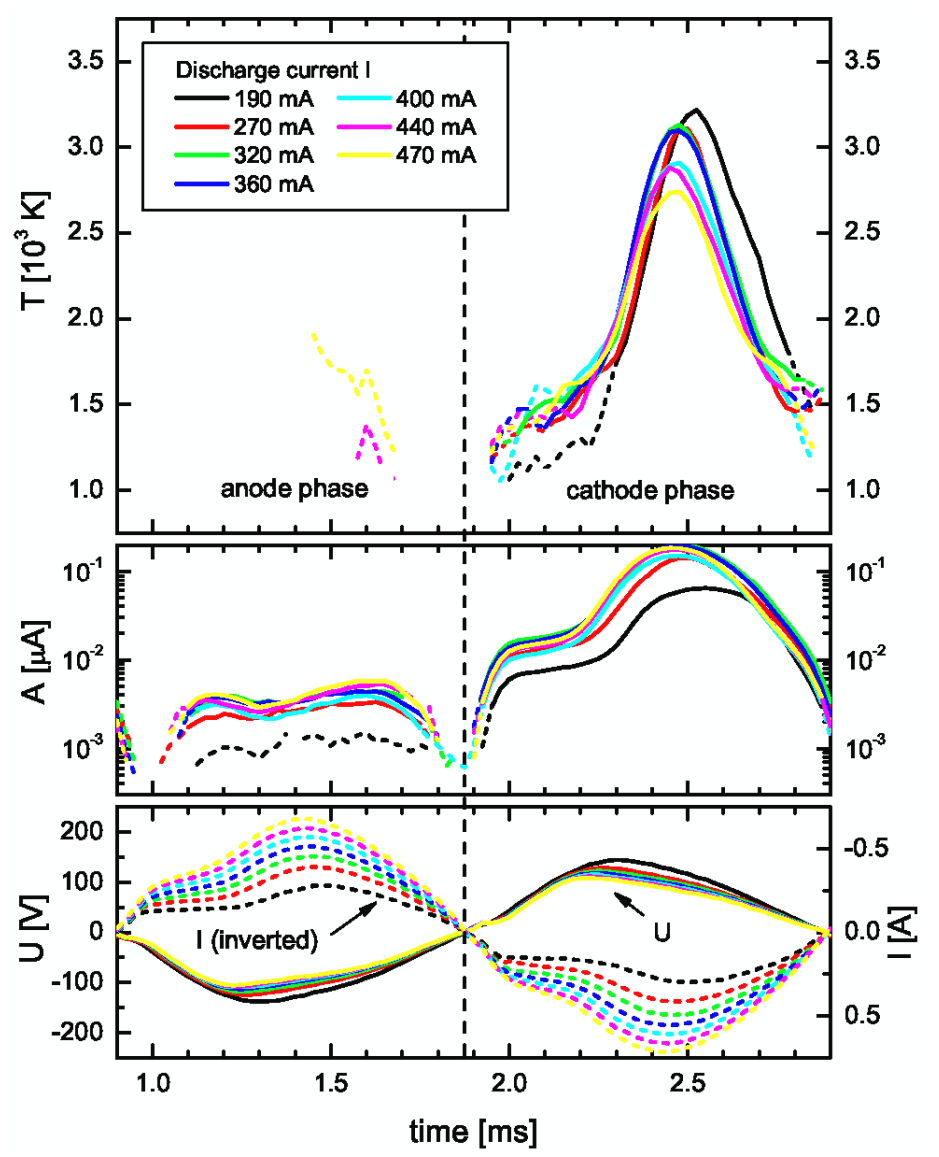

Figure 9. Barium temperature (above), intensity of the barium signal (middle) and voltage/current (below) of a mercury free argon discharge lamp $(\mathrm{p}=2.5$ Torr) for different discharge currents. Barium is presumed sputtered for all currents shown. 


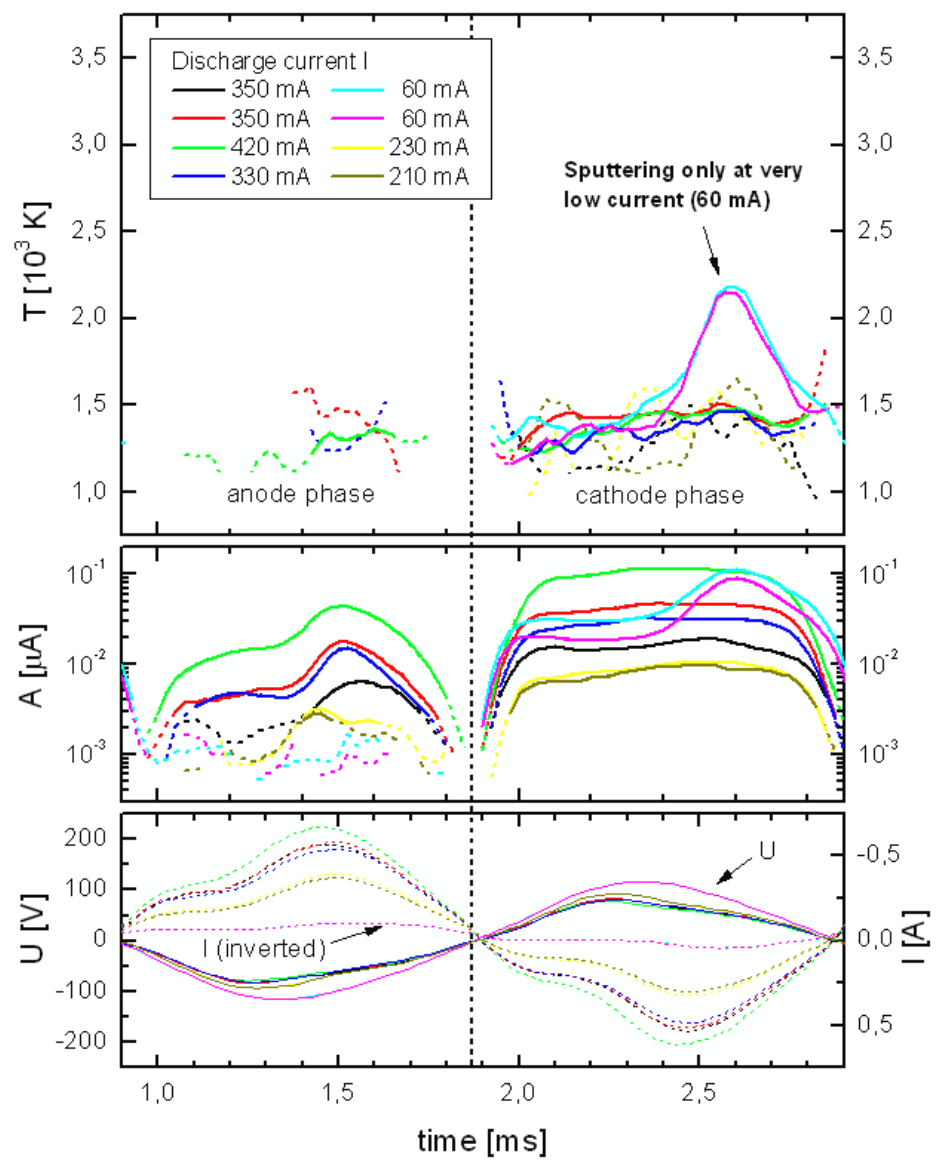

Figure 10. Barium temperature (above), intensity of the barium signal (middle) and voltage/current (below) of a mercury-argon discharge lamp ( $\mathrm{p}=2.5$ Torr) at different discharge currents. Barium is sputtered only for the lowest current of $60 \mathrm{~mA}$. 


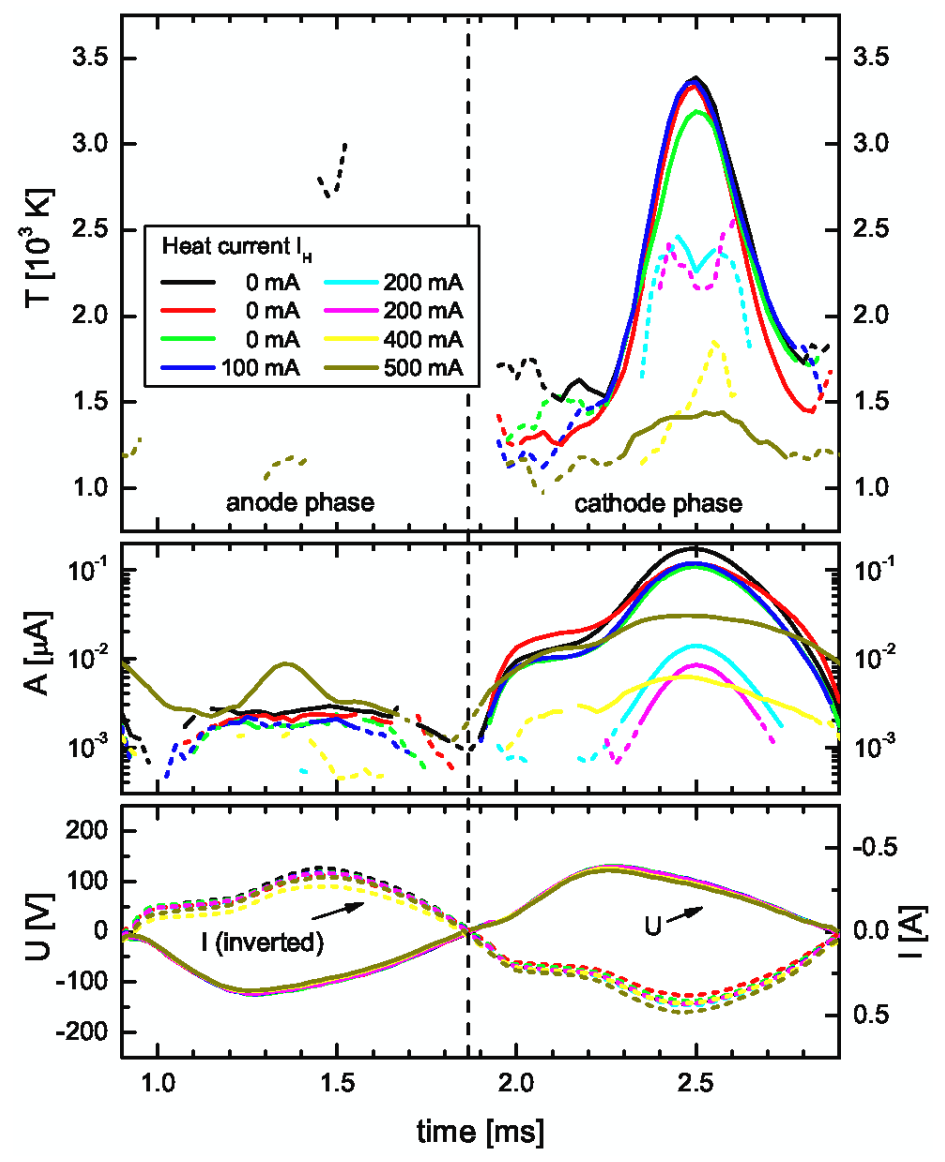

Figure 11. Barium temperature (above), intensity of the barium signal (middle) and voltage/current (below) of a mercury free argon discharge lamp ( $\mathrm{p}=2.5$ Torr) for different heating currents. The discharge current for the different measurements varied slightly in the small range from 240 to $280 \mathrm{~mA}$. 

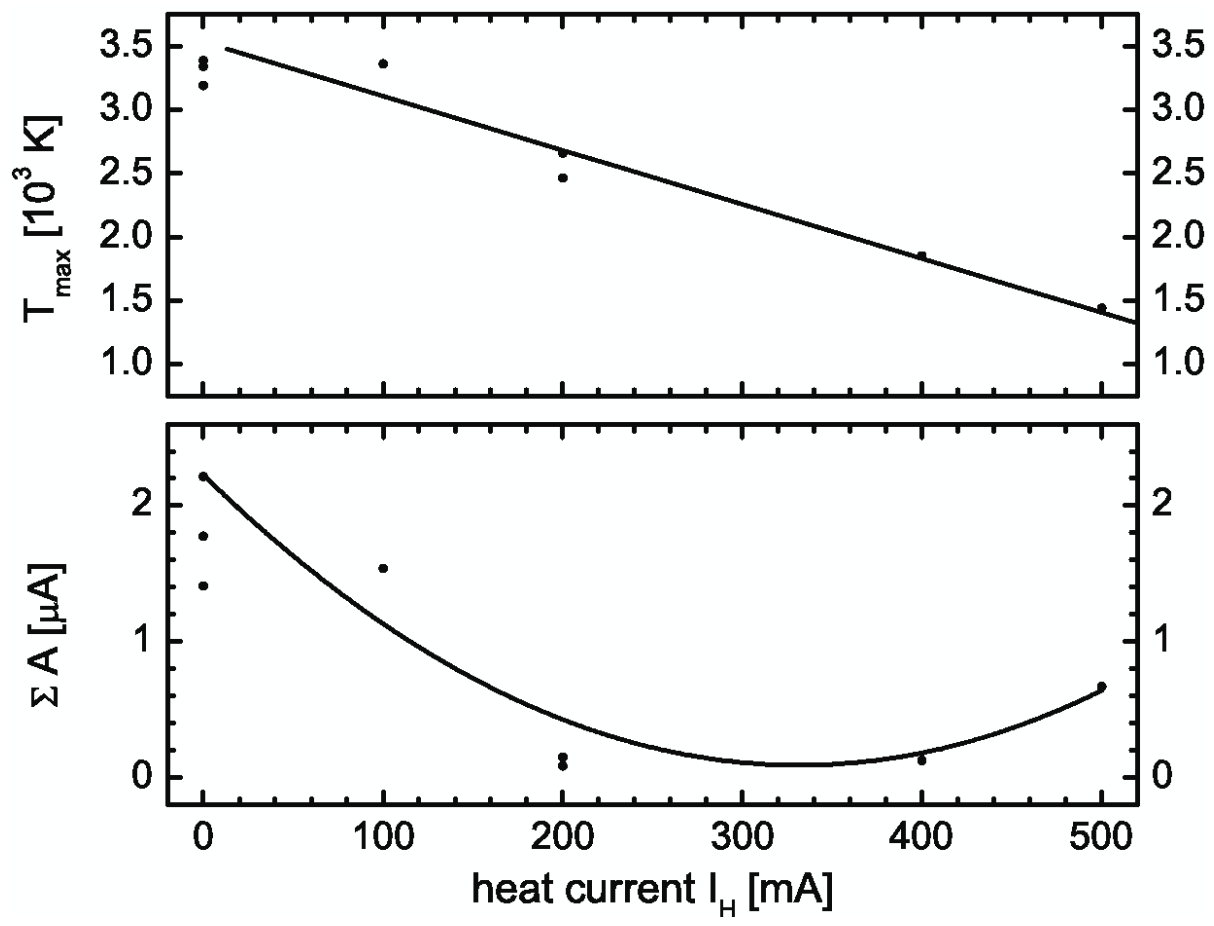

Figure 12. Peak temperature (above) and integrated intensity over the cathode phase of the barium signal (below) of a mercury free $\operatorname{argon}$ discharge lamp $(\mathrm{p}=3.3 \mathrm{mbar})$ at a discharge current of $240 \ldots 280 \mathrm{~mA}$. 

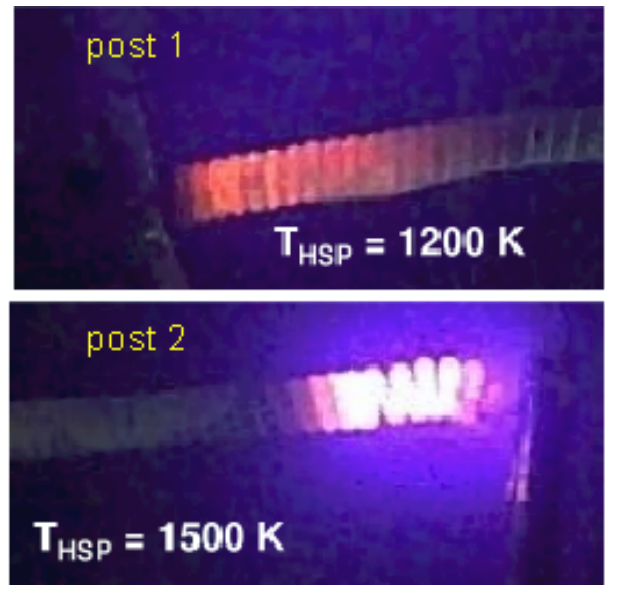

Figure 13. Pictures of hot spots that happened to occur (not simultaneously) on opposite ends (near the support posts) of the same electrode. The upper picture shows an apparently cooler hot spot. This is borne out by FPI and ETD measurements, shown in Figure 14.

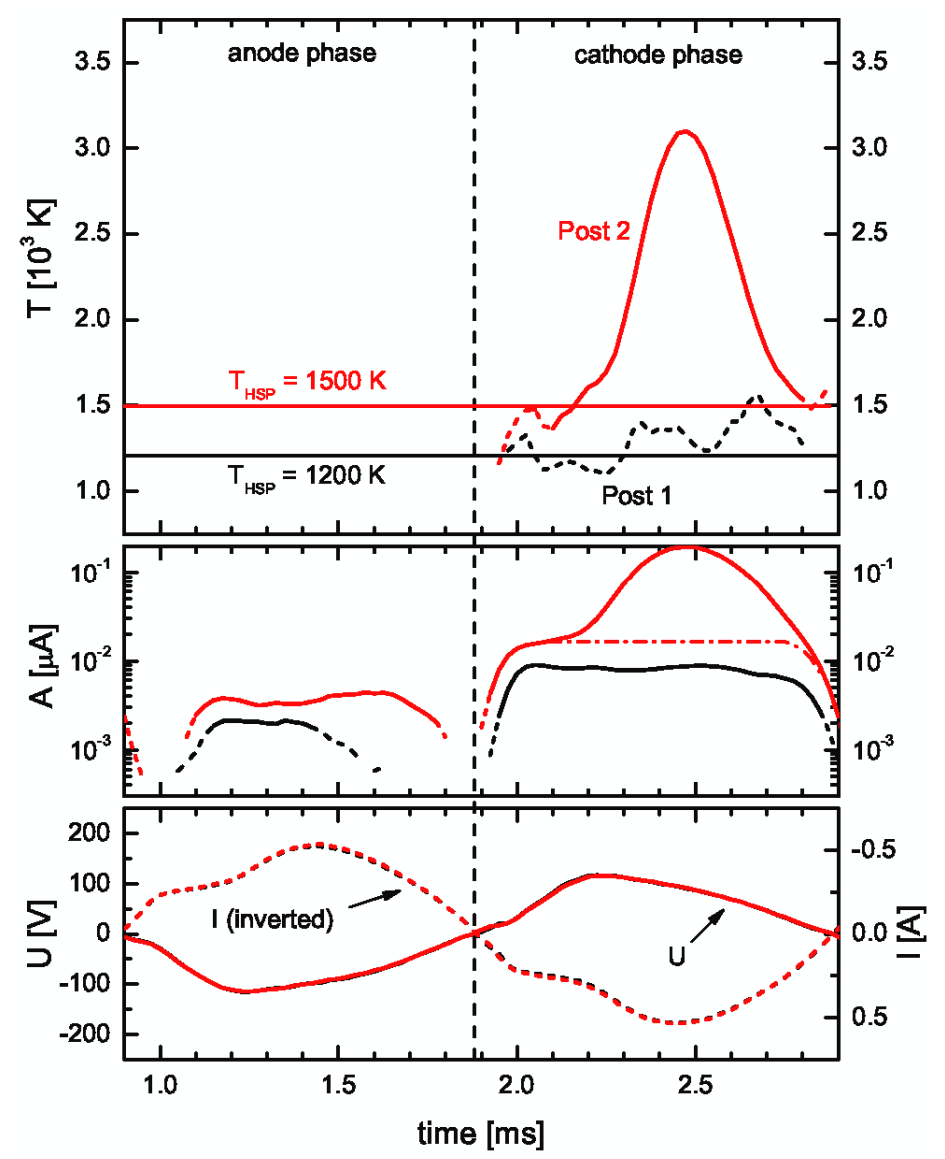

Figure 14. FPI and ETD measurements of the two hot spots depicted in Figure 13. 


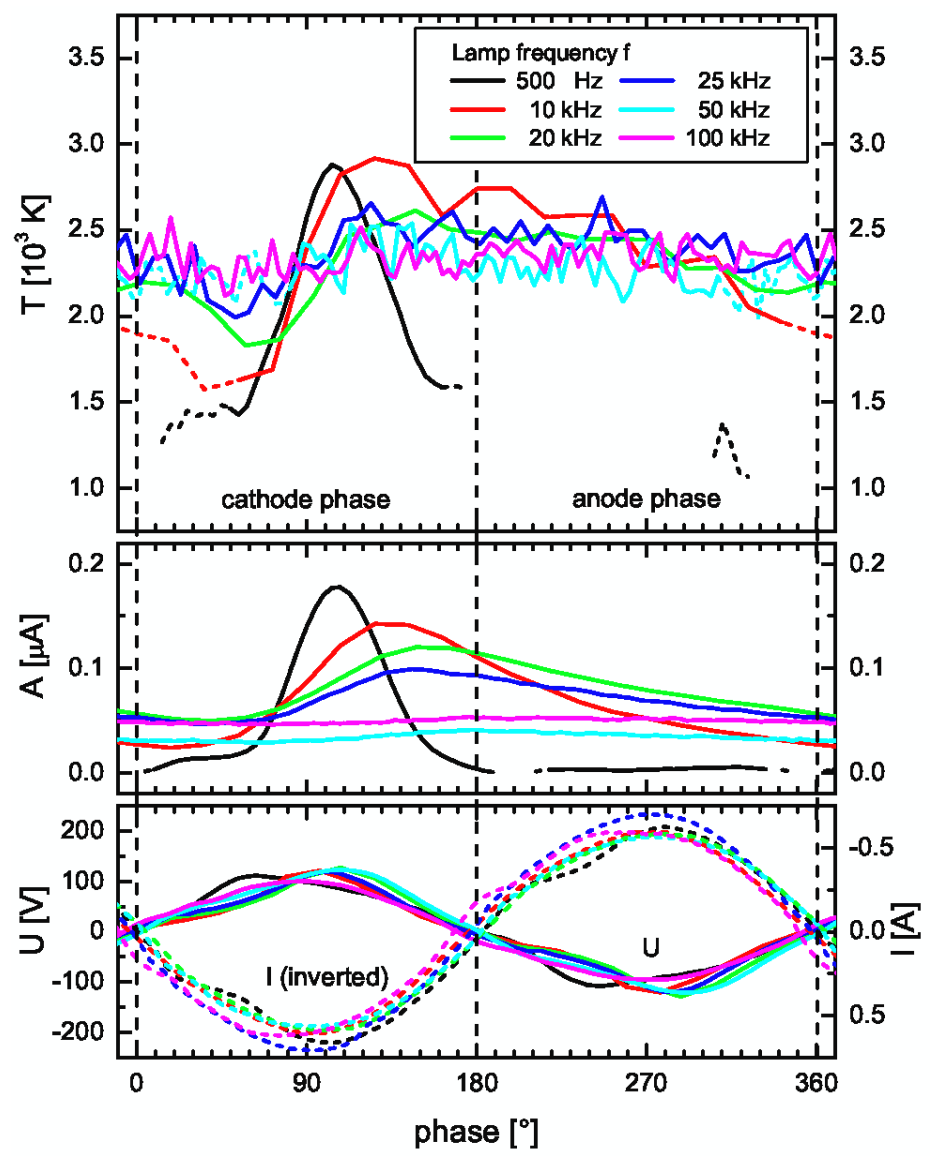

Figure 15. Barium temperature (above), intensity of the barium signal (middle) and voltage/current (below) of a mercury free $\operatorname{argon}$ discharge lamp $(\mathrm{p}=2.5$ Torr) for different frequencies. The discharge current is in the range of 420 to $500 \mathrm{~mA}$. 


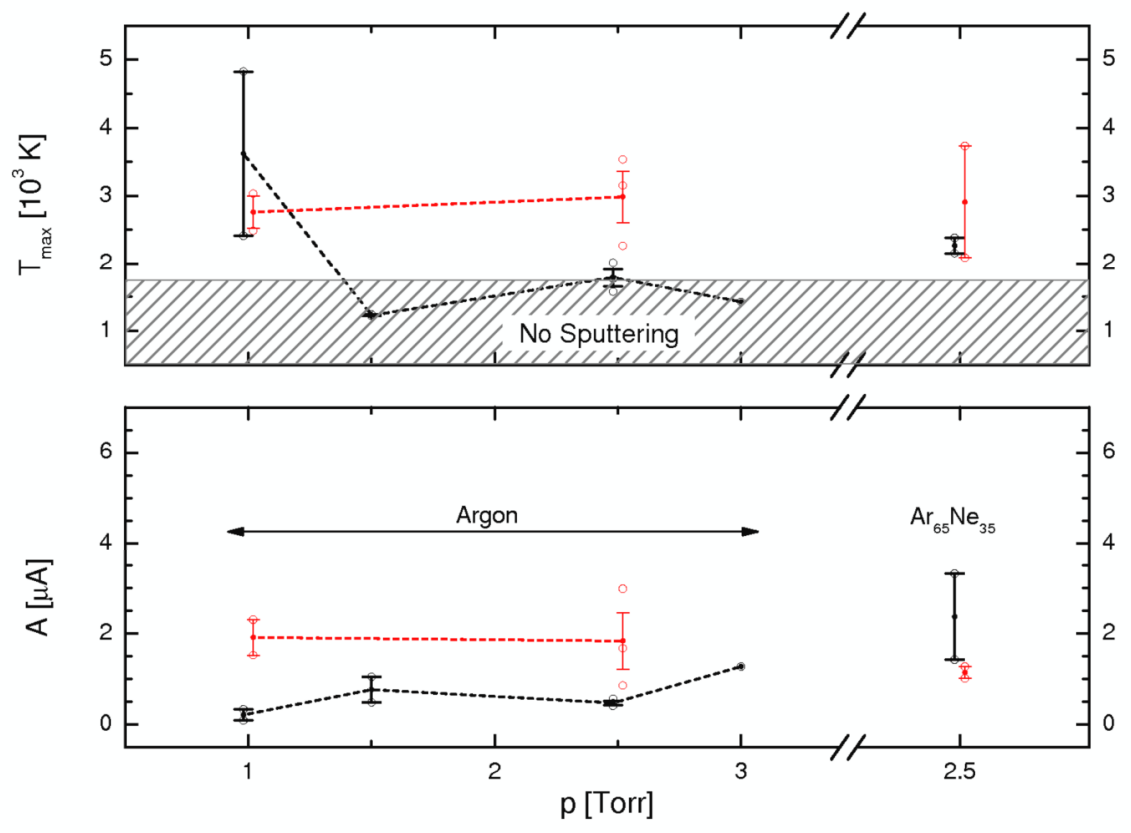

Figure 16. Peak temperature (above) and integrated intensity over the cathode phase of the barium signal (below) of a various number of mercury free (red) and mercury containing (black) discharge lamps at a discharge current of $200 \ldots 200 \mathrm{~mA}$. Each data point represents one lamp.

\section{Conclusions}

A Fabry-Perot interferometer (FPI) based diagnostic has been developed and subsequently used to identify regimes in which barium is sputtered from the electrodes of fluorescent lamps during their steady-state operation. The diagnostic senses the line emission from barium atoms which have been excited in the discharge environment after they are liberated from the electrode. The Fabry-Perot interferometer resolves the spectral width of the line emission, thereby allowing us to infer temperature, since the broadening is primarily due to the Doppler effect.

Interpretation of the data requires a deconvolution with an instrument function, which is the response of the diagnostic to zero-spectral width radiation. In using the diagnostic, it is necessary to have a model for the instrument function and to measure it using a laser. For the model it is necessary to consider the effects of apparent spectral spread due to angular spread of the radiation through the FPI. This is in addition to the instrinsic spread due to the finite finesse (or, equivalently, the finite reflectivity of the mirrors) of the FPI.

In using the diagnostic we have found that under many conditions barium simply evaporates from the thermionicially emitting electrode. However, there are instances when cathode falls are at high enough levels to lead to sputtering. In particular, rare gas discharges exhibit sputtering over a broad range of discharge currents. For low frequency operation (500 Hz reported here), sputtering occurs during the cathode phase, but only after some initial period during which barium is evaporated. Sputtering can be eliminated by the application of large enough auxiliary electrode heating currents. Mercury-rare gas discharges exhibit sputtering only at sufficiently low discharge currents. These results are consistent with established understandings of the plasmaelectrode interface of low pressure, thermionic (non-thermal) discharges. 


\section{Appendix A. Instrument Function}

\section{Appendix A.1. Derivation}

Here we establish the system response function (ratio of transmitted to incident power) for general spectral and angular profiles of the incident radiation. The instrument function, the response for a zero-spectral width (Dirac delta) profile, will come out of the derivation.

The well-known FPI transmittance for an incident ray of specific wavelength and angle of incidence, assuming no absorption by the FPI mirrors, is [19]:

$$
T=\frac{1}{1+K \sin ^{2}\left(\frac{\phi}{2}\right)}
$$

where $\phi$ is the phase difference between successive internally-reflected rays that exit the FPI, and $\mathrm{K}$ is the coefficient of finesse. The curve representing $T(\phi)$ has an infinite series of identical peaks with periodicity in $\phi$ of $2 \pi$ radians. The finesse, the ratio of the period to the full-widthat-half-maximum (FWHM) of the peaks, is

$$
F=\frac{\pi}{2 \sin ^{-1}\left(1 / K^{1 / 2}\right)}
$$

It is typical that $K \gg>1$, in which case $F \approx \pi \sqrt{K} / 2$.

For radiation incident at angle $\theta$ with respect to the FPI axis, the phase, $\phi$, is given by [19]:

$$
\frac{\phi}{2}=k L \cos \theta
$$

where $k$ is the magnitude of the wave vector of the radiation, and $L$ is the spacing between the FPI mirrors. Considering the possibility of variations in $\mathrm{k}, \mathrm{L}$, or $\theta$, equation (A.3) can be written as

$$
\frac{\phi}{2}=\left(k_{o}+\Delta k\right)\left(L_{o}+\Delta L\right)\left(1+\frac{(\Delta \theta)^{2}}{2}\right)
$$

where $k_{o}$ and $L_{o}$ are baseline values (assumed constant), and $\Delta k$ and $\Delta L$ are variations from the baselines. $\Delta k$ is associated with the spectral profile of the measured radiation and $\Delta L$ is associated with the movement of the FPI mirrors. The quantity $\Delta \theta<<1$ is considered to be a small variation of the angle of incidence from a zero baseline value (i.e., $\theta_{o}=0$ ). The baseline 
values, $k_{o}$ and $L_{o}$, are arbitrary, however they are chosen as follows. The value $k_{o}=2 \pi / \lambda_{o}$ corresponds to the center wavelength of the radiation of interest $\left(\lambda_{o} \sim 553 \mathrm{~nm}\right)$. Therefore $\Delta k<<k_{o}$ (equivalently, $\Delta v<<v_{o}$, where $v_{o}=c / \lambda_{o}$ ). The value $L_{o}$ is the nominal macroscopic spacing of the mirrors $\left(L_{o} \approx 1 \mathrm{~cm}\right.$ ), but chosen such that $k_{o} L_{o}=N \pi$, where $N$ is an integer. Insofar as the mirrors of a tunable FPI move, at most, by distances of a few wavelengths of the radiation, then $\Delta L<<L_{o}$. Note that $N=L_{o} / L_{F S R}$, where $L_{F S R}=\lambda_{o} / 2$ is the periodicity of the FPI transmission with respect to mirror spacing, for fixed radiation frequency and zero incidence angle.

Multiplying the factors of equation (A.4) and retaining only the lowest (existing) non-zero order terms of the variations gives the following:

$$
\begin{aligned}
\frac{\phi}{2} & \approx \frac{2 \pi}{c}\left[L_{o} \Delta v+v_{o} \Delta L+\frac{1}{2} v_{o} L_{o}(\Delta \theta)^{2}\right] \\
& =\frac{\pi}{v_{F S R}}\left(\Delta v-\Delta v_{F P I}+\Delta v_{\theta}\right)
\end{aligned}
$$

The zero order term is neglected since it is $k_{o} L_{o}=N \pi$, and therefore does not affect the value of the transmittance function (A.1). In (A.5) the following definitions have been made:

- The free spectral range, $v_{F S R}=c / 2 L_{o}$, is the periodicity of the FPI transmission with respect to radiation frequency, for fixed mirror spacing and zero incidence angle. In the present work $v_{F S R}=15 \mathrm{GHz}$. It is noted that $v_{F S R}=v_{o} / N$.

- The effective FPI frequency due to mirror spacing $\Delta v_{F P I}=-\left(v_{o} / L_{o}\right) \Delta L=-\left(v_{F S R} / L_{F S R}\right) \Delta L$. The negative sign in the definition is due to the fact that smaller mirror spacing is associated with larger radiation frequencies, and vice-versa.

- The frequency shift due to angle of incidence variation $\Delta v_{\theta}=\left(v_{o} / 2\right) \Delta \theta^{2}$, or, equivalently, $\Delta v_{\theta} / v_{F S R}=\frac{N}{2} \Delta \theta^{2}$.

The FPI response function, $R\left(\Delta v_{F P I}\right)$, is now obtained by inserting (A.5) into (A.1), multiplying by the spectral profile, $S(\Delta v)$, and angular profile, $\Psi(\Delta \theta)$, of the incident radiation, and integrating over radiation frequency and angle. For the angular profile we assume that the radiation power is uniformly distributed within a cone of angles centered at zero and with maximum equal to $\theta_{m}$. Normalized to unity, the angular profile is

$$
\Psi(\Delta \theta)=\frac{1}{\pi \theta_{m}^{2}}\left\{\begin{array}{cc}
2 \pi \Delta \theta, & \Delta \theta \leq \theta_{m} \\
0, & \Delta \theta>\theta_{m}
\end{array}\right.
$$

The FPI response function is

$$
R\left(\Delta v_{F P I}\right)=\int_{0}^{\infty} d(\Delta v) S(\Delta v) I\left(\Delta v_{F P I}-\Delta v\right)
$$


where I is given by

$$
I(\Lambda)=\frac{1}{\pi \theta_{m}^{2}} \int_{0}^{\theta_{m}} \frac{d(\Delta \theta) 2 \pi \Delta \theta}{1+K \sin ^{2}\left[\pi\left(\Lambda+\frac{N}{2}(\Delta \theta)^{2}\right)\right]}
$$

and $\Lambda=\left(\Delta v_{F P I}-\Delta v\right) / v_{F S R}$.

The function $I(\Lambda)$ is recognized as the instrument profile. The integral in (A.8) can be evaluated analytically [20] with the following result: ${ }^{* * *}$

$$
I(\Lambda)=\frac{1}{\pi F_{\theta}^{-1}}\left(\frac{\tan ^{-1}\left\{\sqrt{1+K} \tan \left[\pi\left(\Lambda+F_{\theta}^{-1}\right)\right]\right\}-\tan ^{-1}\{\sqrt{1+K} \tan [\pi \Lambda]\}}{\sqrt{1+K}}\right)
$$

where

$$
F_{\theta}^{-1}=\frac{N}{2} \theta_{m}^{2}=\frac{\Delta v_{\theta_{m}}}{v_{F S R}}
$$

The quantity $F_{\theta}$ can be interpreted as an effective finesse due to angular spread (it is therefore desirable to have $F_{\theta}<<F$ ). It is noted that each arctangent function that appears in (A.9) should be evaluated on the same branch as the argument of the corresponding tangent function that appears within each [20].

Equation (A.9) is the general form of the instrument profile for the present work. However, we also consider the special case of perfectly collimated radiation incident normal to the FPI. The instrument profile for perfectly collimated radiation is obtained by expanding the right-hand-side of (A.9) with respect to $\theta_{m}$ (or $F_{\theta}^{-1}$ ) and setting $\theta_{m}=0$ (or $F_{\theta} \rightarrow \infty$ ). Since the denominator of (A.9) contains $F_{\theta}^{-1}$, it is necessary to expand the numerator to first order to obtain the zero order of the complete expression. The result is

$$
I_{\text {coll }}(\Lambda)=\frac{1}{1+K \sin ^{2}[\pi \Lambda]}
$$

It is straightforward to show that this is same expression that would have been obtained by considering a delta function angular profile, $\Psi(\Delta \theta)=\delta(\Delta \theta)$. Equation (A.11), is the wellknown standard instrument function for an FPI, an Airy function [19].

${ }^{* * * *}$ The substitution $x=\Lambda+\frac{N}{2}(\Delta \theta)^{2}$ puts the integrand in the same form as the integral in the reference. 
A useful approximation can be considered for the collimated instrument function. If $v_{F W H M}<<v_{F S R}$, which is the case in the present work, then $I_{\text {coll }}$ is only significant for small values of $\Lambda$. In this case, the Airy function can be approximated by expanding the sine function in the denominated to first order in $\Lambda$. The result is a Lorentzian function:

$$
I_{c o l l, L n z}(\Lambda)=\frac{1}{1+\pi^{2} K \Lambda^{2}}
$$

As we show in the next section, it turns out that the temperatures derived by using this instrument function are almost the same as those derived using either the "true" collimated (Airy) instrument function (A.11), and even the full instrument function (A.9). Therefore, for most of the data analysis shown in this paper, (A.12) is the instrument function actually employed.

\section{Appendix A.2. Measurement and Application}

The analysis routine performed on simulated data demonstrates the suitability of using the Lorentzian approximation to the collimated instrument function, $\mathrm{I}_{\text {coll,Lnz }}$, instead of either the true collimated, $\mathrm{I}_{\text {coll }}$, or the full instrument function, I. For the simulated data, Gaussian source functions with various temperatures are constructed. The FPI has $15 \mathrm{GHz}$ free spectral range and intrinsic finesse of 30 , and the angular spread of the incident radiation is $1.67 \mathrm{mrad}$. A "true" simulated instrument function based on these parameters is generated using (A.9). A set of simulated data, for all the temperatures considered, is then calculated with (A.7) using the true simulated instrument function and the Gaussian source functions.

The analysis is performed on the simulated data to determine the temperatures. First, for each temperature, three coefficients of finesse are determined by fitting Eqs (A.9) to (A.11), respectively to the simulated instrument function data. Then, at each temperature three response functions are determined by fitting Eq. (A.7) to the simulated data using the three coefficients of finesse. In these fits, the standard deviations of the Gaussian are free parameters and determined from the fits.

Figure A1 shows the results. Part b shows standard deviations versus the assumed temperatures of the source function. The points correspond to those values derived using both collimated instrument functions (x's for the true and +'s for the Lorentzian approximation), as well as the full instrument function (circles). The line is the true (proscribed) value, corresponding to the assumed temperature. It is seen that the true value hardly deviates at all from that calculated using the full instrument function and only slightly when using either collimated instrument function. Part a shows the fractional difference between the values derived from the full and both collimated instrument functions. It is seen that the maximum deviation over the temperature range considered is $\sim 5 \%$ and only slightly more for the Lorentzian approximation. Thus, if temperatures of $\sim 2000 \mathrm{~K}$ are calculated using the Lorentzian collimated instrument function, then the error due to faulty consideration of the instrument function is $\sim 100 \mathrm{~K}$. This would not affect the general conclusion that barium has been sputtered. It is therefore sufficient to use the Lorentzian approximation to the true collimated instrument function. 


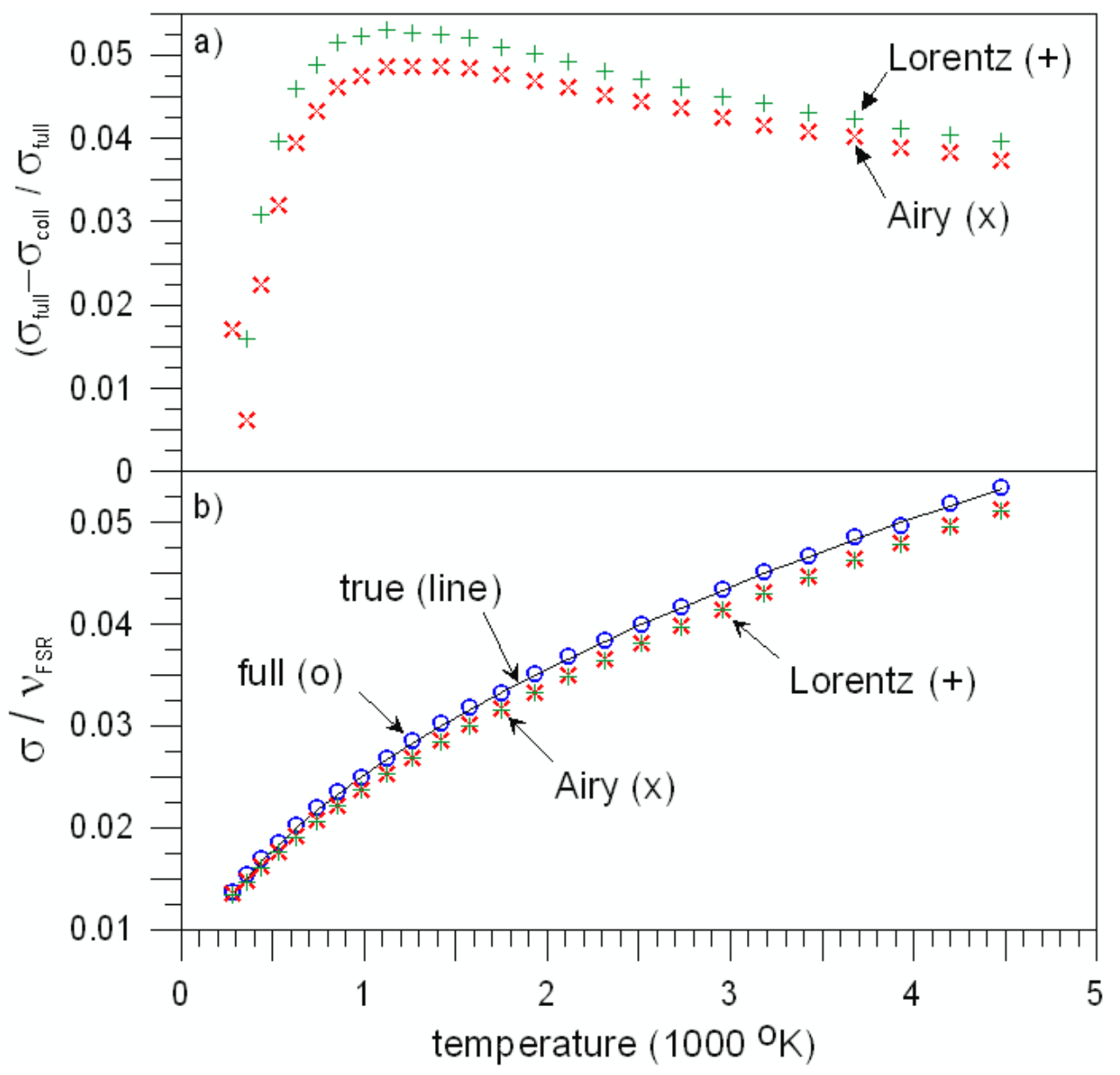

Figure A1. Results of employing the analysis routine on simulated data. The source function is considered to be Gaussian with various standard deviations $\left(\sigma_{\text {true }}\right.$ ) or temperatures. The FPI has $15 \mathrm{GHz}$ free spectral range and instrinsic finesse of 30, and the angular spread of the incident radation is $1.67 \mathrm{mrad}$. a) Fractional difference between the standard deviations obtained from the analysis using the full ( $\left.\sigma_{\text {full }}\right)$ and the collimated ( $\left.\sigma_{\text {coll }}\right)$ instrument functions. Both the true collimated instrument function and the Lorentzian approximation are considered, as shown. The horizontal axis refers to the assumed temperature of the source function. b) Standard deviations obtained by assuming the true collimated instrument function (x's), the Lorentzian approximation to the true collimated instrument function (+'s), and the full instrument function (circles). Also shown is the true standard deviation (line) corresponding to the temperature.

\section{Appendix B. Cascade Velocity Distribution}

Here, we derive an expression for the velocity distribution corresponding to the cascade energy distribution, discussed in section 3.4, and compare the result to a Gaussian function. This provides some justification for our use of the Gaussian function in the fitting routine used to obtain temperature, as described in sections 3.1 to 3.3. 
Equation (3), which gives the normalized cascade distribution cast in terms of energy, E, can be written in terms of a dimensionless energy parameter $\varepsilon=E / U$ (hereinafter referred to as 'energy') as follows:

$$
f(\varepsilon) d \varepsilon=\frac{2 \varepsilon}{(1+\varepsilon)^{3}} d \varepsilon
$$

We assume the motion of the particles to be isotropic and therefore the velocity distribution, $g(\bar{w})=g(w)$, is a function of the magnitude of the velocity, $w . \bar{w}=\left(w_{x}, w_{y}, w_{z}\right)$ is the normalized velocity: $\bar{w}=\overline{\mathrm{v}} / u$, where $\mathrm{u}$ is defined by the relation $U=\frac{1}{2} m u^{2}$. This implies that $\varepsilon=w^{2}$.

The isotropy assumption implies that the number of particles with energies between $\varepsilon$ and $\varepsilon+d \varepsilon$ must equal the number of particles which, in velocity space, is contained within the spherical shell with radius $w$ and thickness $d w$. That is,

$$
4 \pi w^{2} g(\bar{w}) d w=f(\varepsilon) d \varepsilon
$$

where the vector argument is left in $g(\bar{w})$ to indicate that $g$ is to be evaluated everywhere in velocity space. From this we get

$$
g(\bar{w})=\frac{1}{\pi} \frac{w}{\left(1+w^{2}\right)^{3}}
$$

The distribution for any one (cartesian) component of the velocity (which we can arbitrarily take as $w_{z}$ ), independent of the values of the mutually orthogonal components, is then

$$
h\left(w_{z}\right)=\frac{1}{\pi} \int_{-\infty}^{\infty} d w_{x} \int_{-\infty}^{\infty} d w_{y} \frac{\left(w_{x}^{2}+w_{y}^{2}+w_{z}^{2}\right)^{1 / 2}}{\left(1+w_{x}^{2}+w_{y}^{2}+w_{z}^{2}\right)^{3}}
$$

Making the transformation $w_{x}=\rho \cos \theta$ and $w_{y}=\rho \sin \theta$, integrating over $\theta$ (which does not appear in the integrand at this point), and then substituting $x=+\left(\rho^{2}+w_{z}^{2}\right)^{1 / 2}$ gives us

$$
h\left(w_{z}\right)=2 \int_{\left|w_{z}\right|}^{\infty} \frac{x^{2} d x}{\left(1+x^{2}\right)^{3}}
$$

The integral can be done analytically [21] and we get the following result:

$$
h\left(w_{z}\right)=\frac{\pi}{8}-\frac{\left|w_{z}\right|}{4\left(1+w_{z}^{2}\right)}+\frac{\left|w_{z}\right|}{2\left(1+w_{z}^{2}\right)^{2}}-\frac{1}{4} \tan ^{-1}\left(\left|w_{z}\right|\right)
$$


Figure B1 shows a plot of $h(w)$ versus $w$ (the subscript ' $\mathrm{z}$ ' is now dropped). Also shown is a least squares fit using the Gaussian fitting function $G(w)=A \exp \left(-w^{2} / 2 \sigma_{w}^{2}\right)$ with $A$ and $\sigma_{w}$ as free parameters. It can be seen that the cascade distribution has similar form to the Gaussian but with a somewhat extended tail. It turns out that its standard deviation is infinite. However, the standard deviation of the best fit Gaussian is $\sigma_{w}=0.933$. This corresponds to an energy spread (effective temperature normalized to $\mathrm{U}$ ) of $\sigma_{\varepsilon}=0.871$. Thus, in spite of the extended tail (which is most likely not detected in the data anyway), the standard deviation of the velocity component that is measured is very close to the speed, $\mathrm{u}$, associated with the binding energy, $\mathrm{U}$. The effective temperature is similarly close to the binding energy.

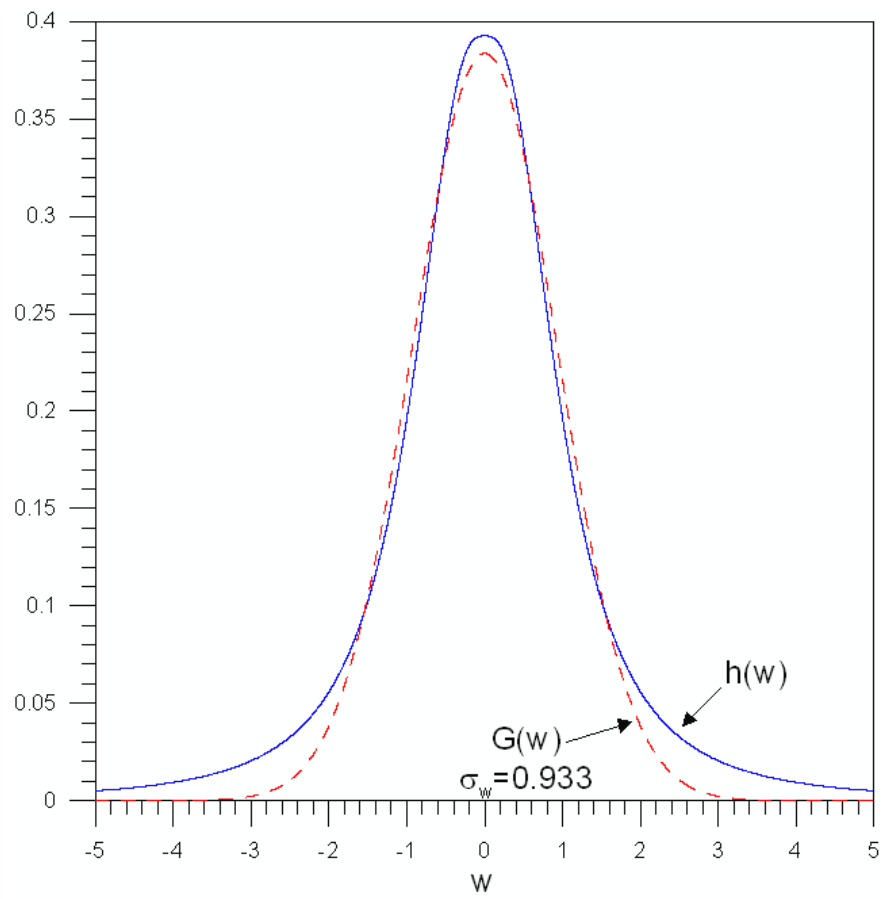

Figure B1. Solid line: cascade distribution, $\mathrm{h}(\mathrm{w})$, for one component, $\mathrm{w}$, of the normalized velocity. Isotropy has been assumed. Dashed line: a least squares Gaussian fit to the cascade distribution. The standard deviation of the cascade distribution is infinite. However, the Gaussian fit has standard deviation of 0.933 . In normalized energy units, $\varepsilon=w^{2}$, where $\varepsilon$ is normalized to the binding energy, $\mathrm{U}$, the standard deviation is 0.871 . Thus, if one fits a Gaussian to data which is intrinsically a cascade distribution, then the "temperature" of the Gaussian should be about $13 \%$ lower than the binding energy of the cascade.

\section{References}

[1] Dang T, Frisk T and Grossman M 2002 Anal Bioanal Chem 373 560-70

[2] Waymouth J 1971 Electric Discharge Lamps (Cambridge, MA: MIT Press) pp 95-102 
[3] Drawert S, Riemann W and Jordanov W Contrib. Plasma Phys. 311991

[4] Garner R 1999 Measurement of barium emitted from thermionic electrodes in steady-state Hg-rare gas discharges Proc. $26^{\text {th }}$ IEEE Int. Conf. on Plasma Science (Monterey, CA, USA) P

[5] Garner R 2007 J. Phys. D: Appl. Phys. 41144010

[6] Hadrath S, Garner R, Lieder G and Ehlbeck J 2007 J. Phys. D: Appl. Phys. 40 6975-81

[7] Hull A 1929 General Electric Review 213-23

[8] Stuart R V \& Wehner G K 1962 J. Appl. Phys. 33 2345-52

[9] Garner R 2005 Time resolved measurements of velocity distributions of sputtered barium in fluorescent lamps Proc. $32^{\text {nd }}$ IEEE Int. Conf. on Plasma Science (Monterey, CA, USA) 3P72.

[10] Garner R 1998 A temperature and emissivity diagnostic for fluorescent lamp electrodes

Proc.8th International Symposia on the Science and Technology of Light Sources

[11] Langmuir I 1916 Phys. Rev. 7 302-30.

[12] Svelto O 1989 Principles of Lasers (New York: Plenum Press) pp 179-84

[13] Smith W J 1990 Modern Optical Engineering $2^{\text {nd }}$ edition (New York: McGraw Hill) p 157

[14] Reif F 1965 Fundamentals of Statistical and Thermal Physics (New York: McGraw Hill)

[15] Gnaser H 1999 Low-Energy Ion Irradation of Solid Surfaces (Berlin: Springer-Verlag) pp 38-54

[16] Raizer Y 1997 Gas Discharge Physics ed J Allen (Berlin: Springer)

[17] Mellisinos A 1966 Experiments in Modern Physics (New York: Academic Press) 526

[18] Hadrath S, Beck M, Garner R, Lieder G and Ehlbeck J 2007 J. Phys. D: Appl. Phys. $40163-$ 67

[19] Hecht E 1987 Optics (Massachusetts: Addison-Wesley) p 370

[20] Selby S (ed) 1973 Standard Mathematical Tables, $21^{\text {st }}$ edn (Cleveland, OH: CRC Press) 441, 410.

[21] Wolfram Mathematica Online Integrator (http://integrals.wolfram.com/index.jsp) 